\title{
Study of naturally occurring radionuclides in the ECHo set-up
}

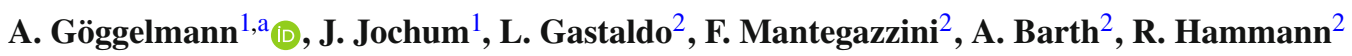 \\ ${ }^{1}$ Physics Institute, University of Tübingen, Tübingen, Germany \\ ${ }^{2}$ Kirchhoff Institute for Physics, Heidelberg University, Heidelberg, Germany
}

Received: 11 November 2021 / Accepted: 7 February 2022 / Published online: 15 February 2022

(C) The Author(s) 2022

\begin{abstract}
The determination of the effective electron neutrino mass by analyzing the end point region of the ${ }^{163} \mathrm{Ho}$ electron capture (EC) spectrum relies on the precise description of the expected ${ }^{163} \mathrm{Ho}$ events and background events. In the ECHo experiment, arrays of metallic magnetic calorimeters, implanted with ${ }^{163} \mathrm{Ho}$, are operated to measure the ${ }^{163} \mathrm{Ho}$ EC spectrum. In an energy range of $10 \mathrm{eV}$ below $Q_{\mathrm{EC}}$, the maximum available energy for the EC decay of about $2.8 \mathrm{keV}$, a ${ }^{163} \mathrm{Ho}$ event rate of the order of $10^{-4} \mathrm{day}^{-1} \mathrm{pixel}^{-1}$ is expected for an activity of $1 \mathrm{~Bq}$ of ${ }^{163} \mathrm{Ho}$ per pixel. This means, a control of the background level in the order of $10^{-5}$ day $^{-1}$ pixel $^{-1}$ is extremely important. We discuss the results of a Monte Carlo study based on simulations, which use the GEANT4 framework to understand the impact of natural radioactive isotopes close to the active detector volume in the case of the ECHo-1k set-up, which is used for the first phase of the ECHo experiment. For this, the ECHo-1k set-up was modeled in GEANT4 using the proper geometry and materials, including the information of screening measurements of some materials used in the ECHo-1k set-up and reasonable assumptions. Based on the simulation and on assumptions, we derive the expected background around $Q_{\mathrm{EC}}$ and give upper limits of tolerable concentrations of natural radionuclides in the set-up materials. In addition, we compare our results to background spectra acquired in detector pixels with and without implanted ${ }^{163} \mathrm{Ho}$. We conclude that typical concentration of radioactive nuclides found in the used materials should not endanger the analysis of the endpoint region of the ${ }^{163} \mathrm{Ho}$ EC spectrum for an exposure time of half a year.
\end{abstract}

\section{Introduction}

In the ECHo experiment, the ${ }^{163} \mathrm{Ho}$ electron capture (EC) spectrum is measured using low temperature metallic mag-

a e-mail: alexander.goeggelmann@uni-tuebingen.de (corresponding author) netic calorimeters (MMCs), with ${ }^{163} \mathrm{Ho}$ enclosed in the absorbers via ion implantation [1]. The biggest effect of a finite electron neutrino mass on the spectrum can be found in the high energy part, just below the maximum energy available for the decay $Q_{\mathrm{EC}} \simeq 2.8 \mathrm{keV}$, which corresponds to the mass difference between the parent atom ${ }^{163} \mathrm{Ho}$ and daughter atom ${ }^{163}$ Dy [2]. The ${ }^{163}$ Ho spectrum is characterized by several resonances, one for each of the excited states in which the daughter atom can be left and continuous structures related to the emission of X-rays and electrons to the continuum [3,4] (Fig. 1). Only a small fraction of the order of $10^{-9}$ of all decays are located in the region of interest (ROI) of $10 \mathrm{eV}$ below $Q_{\mathrm{EC}}$. The set-up used for the simulations based on the ECHo-1k design, which consists of an array of 72 MMCs based detector pixels [5]. Accordingly, a count rate of approximately $8 \cdot 10^{-5}$ day $^{-1}$ pixel $^{-1}$ is expected in the ROI.

\section{Detection mechanism of MMCs}

The energy $E$ deposited by a particle in the absorber of a MMC leads to an increase in temperature $\delta T$ of the MMC:

$\delta T \propto \frac{E}{C}$,

where $C$ is the total heat capacity of the MMC. The increase of the temperature of the paramagnetic sensor results in a change of magnetic moments in the sensor material and thus, in a change of magnetization $\delta M \propto \delta T$, which is measured with SQUIDS [6].

\section{Objectives}

We consider five background sources: Unresolved pile-up, electromagnetic noise, muons, co-implanted radioactive isotopes and, the topic of this work, naturally occurring radionuclides. In the ECHo experiment, the background level due to naturally occurring radionuclides and due to cosmic radiation 


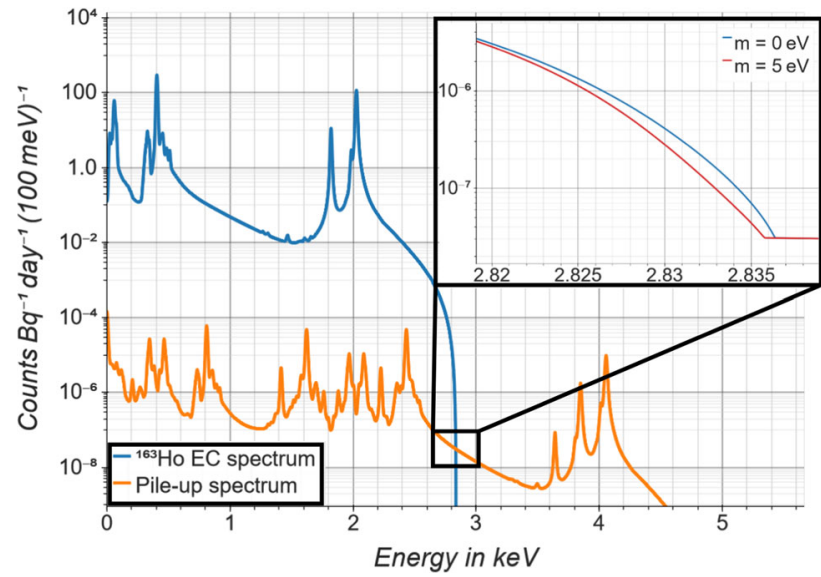

Fig. 1 Expected ${ }^{163}$ Ho EC spectrum (blue solid curve) following [3] and related unresolved pile-up spectrum for a pile-up fraction of $10^{-6}$ (orange solid curve) and considering events with equal and opposite polarity. A Q-value of $2.833 \mathrm{keV}$ and an energy resolution of FWHM $=10 \mathrm{eV}$ are used to calculate the spectra. Inset: The effect of massive neutrinos on the summed spectrum is shown

(mainly muons and secondary particles produced by muons) has to be reduced in a way that the unresolved pile-up remains the highest background contribution in the ROI.

Preliminary Monte Carlo simulations, performed for the detector set-up of the ECHo-1k experiment [1], provided indicative upper limits on the tolerable concentration of radionuclides in the detector and surrounding materials $[7,8]$. Because of the small pixel size of $180 \times 180 \times 10 \mu \mathrm{m}^{3}$, these limits of a few $\mathrm{mBq}$ are relatively high and can easily be fulfilled for most of the common radionuclides. In the following, new updated and more detailed Monte Carlo simulations based on screening measurements on some materials used in the ECHo-1k design are discussed in order to determine the expected background contribution due to the identified radionuclides and to give updated upper limits of tolerable concentrations.

In addition, background spectra measured with ${ }^{163} \mathrm{Ho}-$ loaded and non-loaded pixels are studied and compared to the simulations.

\section{Detector set-up}

A simplified design of the ECHo-1k experimental set-up is used in the simulations as shown in Fig. 2. On top of a silicon ECHo-1k chip [9] of dimension $10 \mathrm{~mm} \times 5 \mathrm{~mm}$ and a thickness of $350 \mu \mathrm{m}, 36 \mathrm{MMC}$ pairs are placed, resulting in 72 pixels positioned in an array of four lines with 18 pixels each (compare to the insert in Fig. 2). The pixels consist of gold absorbers of dimensions $180 \mu \mathrm{m} \times 180 \mu \mathrm{m} \times$ $10 \mu \mathrm{m}$, which are placed directly on top of $\mathrm{Ag}$ : Er sensors of dimensions $168 \mu \mathrm{m} \times 168 \mu \mathrm{m} \times 1.35 \mu \mathrm{m}[9,10]$. In the

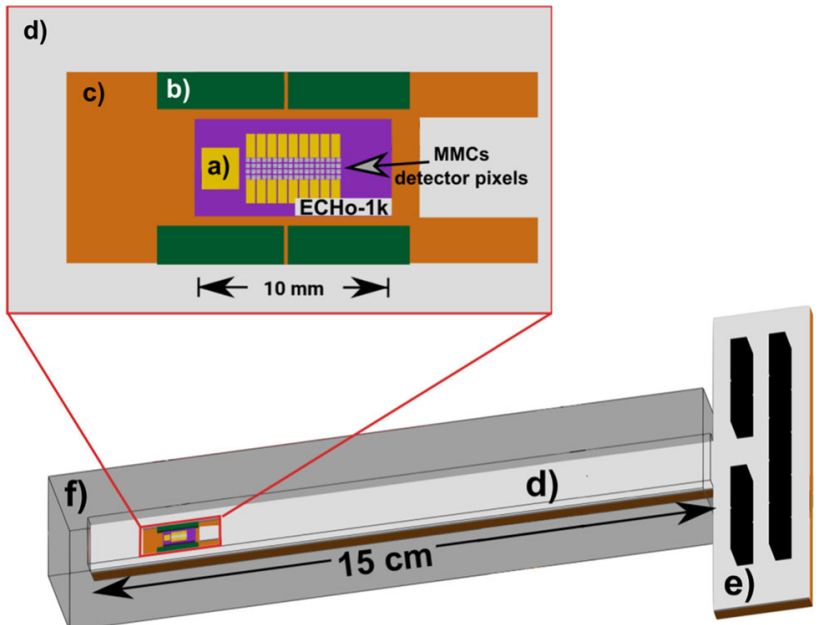

Fig. 2 Simplified design of the ECHo-1k experiment set-up used in the simulation. Inset: The region close to the ECHo- $1 \mathrm{k}$ chip. On the purple-colored silicon ECHo-1k chip, $72 \mathrm{MMCs}$ and large thermalization structures (a) are placed. Next to the ECHo-1k chip, four dc-SQUID chips (b) are located. All five silicon-based chips are directly glued on the copper holder (c). A circuit board (d) covers the full copper holder, except the region next to the ECHo-1k chip. Connectors (e) are placed at a distance of about $15 \mathrm{~cm}$ from the ECHo-1k chip. An aluminum box (f) surrounds the copper holder

middle of the absorbers, thin layers of silver with a thickness of $200 \mathrm{~nm}$ and a surface of about $150 \mu \mathrm{m} \times 150 \mu \mathrm{m}$ are placed as host material for ${ }^{163} \mathrm{Ho}$. Close to the pixels, relatively large gold structures are positioned (Fig. 2a). Four silicon chips, representing the dc-SQUID chips (Fig. 2b), with dimensions of $6 \mathrm{~mm} \times 2.5 \mathrm{~mm}$ and thickness of $350 \mu \mathrm{m}$, are positioned close to the ECHo-1k chip (Fig. 2, insert). The circuit board $^{1}$ (Fig. 2d) covering the copper T-shaped support (called copper holder, Fig. 2c) is modeled as a $0.7 \mathrm{~mm}$ thick layer of a bisphenol-based epoxy resins. The copper holder, obtained by a larger annealed oxygen free high conductivity (OFHC) copper piece, has a thickness of $0.7 \mathrm{~cm}$. The T-shape is required in order to have the ECHo-1k chip and SQUID chips at a distance of about $13 \mathrm{~cm}$ from the aperture of an aluminum tube used as superconducting shield (Fig. 2f). The circuit board has a gap, so that the five silicon chips can directly be glued on top of a copper holder. A rectangular aluminum tube of $2.5 \mathrm{~cm}$ (width) $\times 0.9 \mathrm{~cm}$ (height) and a length of $15 \mathrm{~cm}$ surrounds the set-up. The wall thickness of the aluminum tube is $3 \mathrm{~mm}$ for the bottom and top side and $10.3 \mathrm{~mm}$ for the other sides. Last, nine carbon-based connectors ${ }^{2}$ with a density of $1 \mathrm{~g} \mathrm{~mol}^{-1}$ are placed at a distance of about $15 \mathrm{~cm}$ away from the ECHo-1k chip (Fig. 2e).

\footnotetext{
${ }^{1}$ Multi Leiterplatten GmbH, Brunnthaler Str. 2, D-85649 BrunnthalHofolding, Germany.

2 Connector type SHF-108-01-L-D-TH produced by Samtec, 520 ParkEast Boulevard, New Albany, IN 47140, USA.
} 


\section{Background sources different to natural radionuclides}

\subsection{Unresolved pile-up}

The time profile of the voltage signal caused by the absorption of energy in a MMC is defined by the thermodynamical properties of the detector itself. The shape of the signal can be described by an exponential rise and decay. The rise time $\tau_{\mathrm{r}}$ of these pulses, limited by readout bandwidth, is about $500 \mathrm{~ns}$ for MMCs in the ECHo-1k phase, while the decay time is a few ms [9]. The rise time defines approximately the time resolution of the detectors i.a., if two decays occurred in the same MMC within a time interval shorter than $\tau_{\mathrm{r}}$, they will be interpreted as a single signal with an amplitude corresponding to the sum of the energy released in the two decays. Since each detector channel consists of two pixels, which generate signals of opposite polarity, the unresolved pile-up rate is in first approximation given as twice the product of the activity per pixel A (assuming both pixels having the same ${ }^{163} \mathrm{Ho}$ activity) and the time resolution $\tau_{\mathrm{r}}$. Therefore, the count rate is given by $r_{\mathrm{pu}}=2 \cdot A^{2} \cdot \tau_{\mathrm{r}}$. In Fig. 1 , the continuous blue line represents the expected ${ }^{163}$ Ho spectrum, following the description in [3] and using a Q-value of $2.833 \mathrm{keV}$, while the orange line represents the pile-up spectrum. This pileup spectrum was calculated considering a time resolution of $\tau_{\mathrm{r}}=500 \mathrm{~ns}$ and an activity of $1 \mathrm{~Bq}$ per pixel, leading to an unresolved pile-up fraction of $f_{\mathrm{pu}}=2 \cdot A \cdot \tau_{\mathrm{r}}=10^{-6}$. Note that, due to the different polarities of signals generated by the two pixels of the same channel, the total amplitude of a pile-up event can be smaller than the two single signal amplitudes. The pile-up rate in the last $10 \mathrm{eV}$ is about $3 \cdot 10^{-6}$ day $^{-1}$ pixel $^{-1}$.

At this point, we would like to note that a more precise shape of the unresolved pile-up spectrum depends on the data reduction algorithm, in particular in relation to pulse shape analysis, which might be more suitable to discriminate pileup events when the second events have energies above a few hundred $\mathrm{eV}$, as described in [11].

\subsection{Electromagnetic noise}

Electromagnetic noise can couple to the readout chain of the detectors, resulting in additional background signals. These events can easily be rejected with an efficiency of almost $100 \%$ based on their trigger time information[11].

\subsection{Muons}

Cosmogenic muons can interact with the MMCs and the material in their surrounding. Thus, muons can deposit energy directly in the MMCs and/or can generate secondary radiation, which can interact with the detector pixels. It was shown that these types of events can either be recognized with the use of an active muon veto, by searching for coincidence among pixels events and by their pulse shape [12]. Out of $242 \pm 20$ measured pixel-veto coincidences, $194 \pm 12$ events were also coincidences among pixels, leading to an efficiency of about $(80 \pm 8) \%$ of detecting muon induced events by searching for coincidence among pixels.

In addition, by interacting with materials, muons can generate free neutrons, which can activate the materials. In copper, for example, radioactive isotopes of cobalt (like ${ }^{60} \mathrm{Co}$ ) or other radionuclides (like ${ }^{54} \mathrm{Mn}$ ) can be produced with an accumulated activity concentration of up to the order of $\mathrm{mBq} \mathrm{kg}^{-1}$ [13].

\subsection{Co-implanted isotopes}

In the production process of ${ }^{163} \mathrm{Ho}$, several other radioistopes, for example ${ }^{166 m} \mathrm{Ho}$ [14] or ${ }^{170} \mathrm{Tm} /{ }^{171} \mathrm{Tm}$ [15], are produced. In the ECHo experiment, a two-step separation is used to minimize these byproducts. In particular, a chemical separation is used to ensure that no isotopes different to holmium are left over from the production process [15], followed by mass separation of the holmium fraction at the RISIKO facility [16]. With this, a total ${ }^{166 m} \mathrm{Ho} /{ }^{163} \mathrm{Ho}$ ratio below $5 \cdot 10^{-10}$ is achieved in the MMCs.

\section{Natural radionuclides}

The region of interest for the ECHo experiment is below $3 \mathrm{keV}$. Thus, especially low energy radiation, which could be produced directly in or next to the MMCs, has to be investigated. Radionuclides produce mostly low energy particles when decaying via the $\beta$-decay or EC decay. But also, Auger electrons and $\mathrm{X}$-rays resulting from the rearrangement of the atomic shell, appearing by nuclear decays also including $\alpha$ decays, have a probability to have low energies, below a few $\mathrm{keV}$. In the following, the $\beta$-decaying daughters of the ${ }^{238} \mathrm{U}$ chain $^{3}$ and ${ }^{232}$ Th-chain ${ }^{4}$ as well as ${ }^{40} \mathrm{~K}$ are introduced, which represent the most frequent naturally occurring and relevant nuclides for the ECHo experiment. Only particles with energies less than $20 \mathrm{keV}$ and an emission probability higher than $1 \%$ per decay are listed. The following values are taken from [17] and [18]. The performed simulations include all decay modes, allowing therefore to investigate also low energy particles generated in scattering processes by $\alpha$-particles and other energetic particles like $\gamma$ - and $\delta$-radiation.

\footnotetext{
The $\alpha$-decaying daughters of ${ }^{238} \mathrm{U}$ are mainly ${ }^{234} \mathrm{U},{ }^{230} \mathrm{Th},{ }^{226} \mathrm{Ra}$, ${ }^{222} \mathrm{Rn},{ }^{218} \mathrm{Po},{ }^{214} \mathrm{Po}$ and ${ }^{210} \mathrm{Po}$.

${ }^{4}$ The $\alpha$-decaying daughters of ${ }^{232} \mathrm{Th}$ are ${ }^{228} \mathrm{Th},{ }^{224} \mathrm{Ra},{ }^{220} \mathrm{Rn},{ }^{216} \mathrm{Po}$ and ${ }^{212}$ Po.
} 


\section{$4.1{ }^{238}$ U-chain}

${ }^{238} \mathrm{U}$ can be found in the earth crust with an abundance of about $3 \mathrm{mg}$ per $\mathrm{kg}$ [19] and thus, it also occurs in concrete and metals. The most prominent $\alpha$-decaying daughter of ${ }^{238} \mathrm{U}$ is the gaseous ${ }^{222} \mathrm{Rn}$, which can be accumulated in indoor air with concentrations of several tens of Bq per $\mathrm{m}^{3}$ [20]. Further, radon atoms can be accumulated on surfaces and so, the ${ }^{222} \mathrm{Rn}$ daughters can be implanted in the detector materials due to the recoil after the $\alpha$-decays. For example, concentrations of ${ }^{210} \mathrm{~Pb}$ and ${ }^{210} \mathrm{Po}$ of the order of $10^{-4} \mathrm{mBq} \mathrm{cm}^{-2}$ can be accumulated on the surface of OFHC copper [21].

${ }^{234} \mathrm{Th}$ decays with a Q-value of $274 \mathrm{keV}$ to ${ }^{234} \mathrm{~Pa}$. The half life is 24.1 days. The daughter is always in an excited state of $74 \mathrm{keV}(78 \%), 166 \mathrm{keV}(\sim 20 \%)$ or $187 \mathrm{keV}(\sim 2 \%)$. The corresponding mean energy of the electron is about $54 \mathrm{keV}$ (78\%), $28 \mathrm{keV}(\sim 20 \%)$ or $22 \mathrm{keV}(\sim 2 \%)$ respectively. Protactinium L X-rays of energies between $11 \mathrm{keV}$ and $21 \mathrm{keV}$ and protactinium $\mathrm{L}$ Auger electrons with energies between $6 \mathrm{keV}$ and $21 \mathrm{keV}$ occur each in about $8 \%$ of all decays. In addition, a $11 \mathrm{keV}$ conversion electron (CE) from the L-shell can be emitted in about $4 \%$ of all decays.

${ }^{234} \mathrm{~Pa}$ decays with a Q-value of $2194 \mathrm{keV}$ to ${ }^{234} \mathrm{U}$ with a half life of 6.7 hours. Many transitions can occur, the $\beta$ particles have always relatively large energies ( $>30 \mathrm{keV}$ ) due to the related high Q-value. The probability for emitting uranium L X-rays of energies between $11 \mathrm{keV}$ and $21 \mathrm{keV}$ and uranium $\mathrm{L}$ Auger electrons with energies between $6 \mathrm{keV}$ and $21 \mathrm{keV}$ are very high, they are emitted in about $88 \%$ of all decays. Conversion electrons from the K-shell with energies of $16 \mathrm{keV}(3.9 \%)$ and $19 \mathrm{keV}(1 \%)$ can also be emitted.

${ }^{214} \mathrm{~Pb}$ has a Q-value of $1018 \mathrm{keV}$ and a half life of about 27 minutes. With a probability of about $89 \%$, the daughter nuclide ${ }^{214} \mathrm{Bi}$ can be in four possible excited states of $295 \mathrm{keV}(40 \%), 352 \mathrm{keV}(46 \%), 534 \mathrm{keV}(1 \%)$ and $839 \mathrm{keV}$ ( $\sim 3 \%$ ). The mean energy of the $\beta$-particles corresponding to the decay to the $839 \mathrm{keV}$ state is about $50 \mathrm{keV}$. Bismuth Xrays with energies between 9 and $16 \mathrm{keV}$ occur in about $12 \%$ and the probability that $5 \mathrm{keV}$ to $16 \mathrm{keV}$ bismuth L Auger electrons occur is about $19 \%$.

${ }^{214} \mathrm{Bi}$ decays mostly ( $\sim 99.98 \%$ ) to ${ }^{214} \mathrm{Po}$. The half life of this decay is about 20 minutes with a Q-value of about $3270 \mathrm{keV}$. After the decay, the daughter nuclide is in the ground state in about $19 \%$ of all decays. As in the case of ${ }^{234} \mathrm{~Pa}$, the energy of the emitted $\beta$-particles is large because of the high Q-value. Nearly no low energy particles are expected, only polonium L Auger electrons with energies between $5 \mathrm{keV}$ and $17 \mathrm{keV}$ in about $1 \%$ of all decays.

${ }^{210} \mathrm{~Pb}$ has a half life of about 22 years and a Q-value of about $63 \mathrm{keV}$. In about $84 \%$, the daughter ${ }^{210} \mathrm{Bi}$ is in an excited state with an energy of $47 \mathrm{keV}$. With a probability of about $23 \%$, bismuth $\mathrm{X}$-rays with energies between $9 \mathrm{keV}$ and
$16 \mathrm{keV}$ are emitted. Bismuth L Auger electrons with energies between $5 \mathrm{keV}$ and $16 \mathrm{keV}$ occur in about $35 \%$ of all decays.

${ }^{210} \mathrm{Bi}$ has a half life of about 5 days and a Q-value of about $1160 \mathrm{keV}$. The daughter nuclide is always in the ground sate.

\section{$4.2^{232}$ Th-chain}

${ }^{232} \mathrm{Th}$ can be found in the earth crust with an abundance of about $10 \mathrm{mg}$ per $\mathrm{kg}$ [19] and hence, ${ }^{232} \mathrm{Th}$ and its daughters also occur in metals. As ${ }^{238} \mathrm{U},{ }^{232} \mathrm{Th}$ has also gaseous daughters, however these and their daughters only have short lifetimes. Thus, these can not be accumulated very well on surfaces after the surface is no more exposed to air.

${ }^{228} \mathrm{Ra}$ decays to ${ }^{228} \mathrm{Ac}$ with a half life of about 6 years and a Q-value of about $46 \mathrm{keV}$. The daughter nuclide can be in excited states of about $6 \mathrm{keV}(50 \%), 20 \mathrm{keV}(20 \%)$ or $33 \mathrm{keV}(30 \%)$. This corresponds to small mean energies of the emitted electrons of about $3 \mathrm{keV}$ to $10 \mathrm{keV}$. Due to the small excitation levels of a few keV, low energy gamma radiation can be emitted. Gamma radiation occurs with a probability of about $3 \%$ with energies in the range of $13 \mathrm{keV}$ to $16 \mathrm{keV}$. Actinium L X-rays with energies of $11 \mathrm{keV}$ to $20 \mathrm{keV}$ occur in about $1 \%$ of all decays, as well as $6 \mathrm{keV}$ to $20 \mathrm{keV}$ actinium L Auger electrons. In addition, conversion electrons with energies around $2 \mathrm{keV}(45 \%), 5 \mathrm{keV}$ to $10 \mathrm{keV}$ $(29 \%)$ and $11 \mathrm{keV}$ to $26 \mathrm{keV}(12 \%)$ are emitted.

${ }^{228} \mathrm{Ac}$ further decays to ${ }^{228} \mathrm{Th}$. The Q-value is about $2124 \mathrm{keV}$ and the half life about 6 hours. Almost in any transition, a relatively high energy electron $(>30 \mathrm{keV})$ is emitted and the daughter nuclide is in an excited state. Compared to the other decays, the probability of about $33 \%$ of 11 to $20 \mathrm{keV}$ thorium L X-rays is quite high, as well as the probability of $6 \mathrm{keV}$ to $20 \mathrm{keV}$ thorium L Auger electrons of about $37 \%$.

${ }^{212} \mathrm{~Pb}$ has a half life of about 11 hours and decays with a Qvalue of about $574 \mathrm{keV}$ to ${ }^{212} \mathrm{Bi} .{ }^{212} \mathrm{Bi}$ can be in three states: The ground state $(\sim 12 \%)$, an excited state of an energy of about $239 \mathrm{keV}(\sim 83 \%)$ or of about $415 \mathrm{keV}(\sim 5.2 \%)$. $5 \mathrm{keV}$ to $16 \mathrm{keV}$ bismuth $\mathrm{L}$ auger electrons are emitted in about $22 \%$ of all decays, while $9 \mathrm{keV}$ to $16 \mathrm{keV}$ bismuth $\mathrm{L}$ $\mathrm{X}$-rays occur with a probability of about $14 \%$.

${ }^{212} \mathrm{Bi}$ is special, compared to the other discussed isotopes. The probability of $\beta^{-}$-decay is only about $64 \%$, while $36 \%$ of the decay are an $\alpha$-decay. The half life is about 61 minutes and in case of the $\beta$-decay, the Q-value is about $2252 \mathrm{keV}$. The daughter nuclide ${ }^{212} \mathrm{Po}$ is in the ground state in about $86 \%$ of all $\beta$-decays and in an excited state with an energy between $700 \mathrm{keV}$ and $1805 \mathrm{keV}$ in $14 \%$. X-ray and Auger electrons occur only in case of the $\alpha$-decaying branch. The probability for these emissions after a $\beta$-decay is less than $0.5 \%$. Thus, thallium L X-rays with energies of $9 \mathrm{keV}$ to $15 \mathrm{keV}$ are emitted in about $7 \%$ of all decays and thallium $\mathrm{L}$ Auger electrons with energies between $5 \mathrm{keV}$ and $15 \mathrm{keV}$ in about $12 \%$. 
${ }^{208} \mathrm{Tl}$ decays to ${ }^{208} \mathrm{~Pb}$ with a half life of about 3 minutes and a high Q-value of about $5000 \mathrm{keV}$. After the decay, the daughter nuclide is in one of 15 possible excited states in the range of $3197 \mathrm{keV}$ to $4480 \mathrm{keV}$. $9 \mathrm{keV}$ to $16 \mathrm{keV}$ lead L X-ray occur in about $3 \%$ and lead $\mathrm{L}$ Auger electrons with energies of $5 \mathrm{keV}$ to $16 \mathrm{keV}$ in about $5 \%$.

\section{$4.3{ }^{40} \mathrm{~K}$}

${ }^{40} \mathrm{~K}$ is present in natural potassium with an abundance of $0.0117 \%$ [17]. Its half life is $1.277 \mathrm{~Gy}$ and it has two decay branches. It decays to ${ }^{40} \mathrm{Ca}$ via a $\beta^{-}$process $(\sim 89 \%)$ and a Q-value of around $1311 \mathrm{keV}$ or to ${ }^{40} \mathrm{Ar}$ via electron capture (EC) decay or $\beta^{+}$-decay $(\sim 11 \%)$ and a Q-value of about $1505 \mathrm{keV}$. While the daughter ${ }^{40} \mathrm{Ca}$ nucleus is always in the ground state, the ${ }^{40} \mathrm{Ar}$ nucleus is always in an excited state of $1461 \mathrm{keV}$. In case of the $\mathrm{EC} / \beta^{+}$-decay, $\operatorname{argon} \mathrm{K} \mathrm{X}$-ray with energies around $3 \mathrm{keV}$ are emitted with a probability of about $10 \%$, argon $\mathrm{L}$ Auger electrons with energies of about $300 \mathrm{eV}$ with a probability of about $145 \%$ and argon K Auger electrons with energies between $2.5 \mathrm{keV}$ and $3.2 \mathrm{keV}$ in about $130 \%^{5}$ of all $\mathrm{EC} / \beta^{+}$-decays.

\section{Screening measurement}

In order to estimate the influence of naturally occurring radionuclides in the experimental set-up, screening measurements were performed to identify the presence of radionuclides in the materials used for the detector setup, as well as parts of the cryostat. The screening measurements were conducted in the shallow underground laboratories at Tübingen with a shielding against cosmogenic radiation of about $16 \mathrm{~m}$.w.e. [22]. The sensitivity of the used high purity germanium detector [23] on radioactive contamination is mainly limited by the internal contamination of the detector itself. Dominant internal contamination features of the background spectrum correspond to about $15 \cdot 10^{-4}$ counts s $^{-1}$ of the ${ }^{40} \mathrm{~K} 1460 \mathrm{keV}$ line, $12 \cdot 10^{-4}$ and $18 \cdot 10^{-4}$ counts s$^{-1}$ for the ${ }^{208} \mathrm{Tl} 2615 \mathrm{keV}$ and $583 \mathrm{keV}$ lines respectively and $30 \cdot 10^{-4}$ counts s$^{-1}$ of the $239 \mathrm{keV}^{212} \mathrm{~Pb}$ line. If no larger rates would be measured for the tested samples, an upper limit, the $95 \%$ C.L., is calculated by using the internal limitations.

The screened samples are $50 \mathrm{~g}$ of cryostat copper used as high thermal conductivity holder for the detector set-up indicated with (c) in Fig. 2, a batch of 15 connectors ((e) in Fig. 2) with a total weight of $7.5 \mathrm{~g}$ and a printed circuit board with a weight of $13.63 \mathrm{~g}((\mathrm{~d})$ in Fig. 2). The results of the screening measurement are shown in Table 1 for bulk contaminations

\footnotetext{
${ }^{5}$ More than one of these Auger electrons can be emitted per decay.
}

Table 1 Results of the screening measurement

\begin{tabular}{llll}
\hline Sample & ${ }^{40} \mathrm{~K}$ & ${ }^{232} \mathrm{Th}$ chain & ${ }^{238} \mathrm{U}$ chain \\
\hline Copper & $<0.7$ & $<1.6$ & $<1.3$ \\
Connectors & $<3.1$ & $44 \pm 3$ & $128 \pm 5$ \\
Circuit board & $4 \pm 1$ & $124 \pm 2$ & $82 \pm 3$ \\
\hline
\end{tabular}

The values are given in $\mathrm{Bq} \mathrm{kg}^{-1}$

Table 2 Results of the screening measurement

\begin{tabular}{lll}
\hline Sample & ${ }^{40} \mathrm{~K}$ & ${ }^{210} \mathrm{~Pb}$ chain \\
\hline Copper & $<0.7$ & $<0.3$ \\
Connectors & $<0.4$ & $5.0 \pm 0.2$ \\
Circuit board & $0.4 \pm 0.1$ & $2.4 \pm 0.1$ \\
\hline
\end{tabular}

The values are given in $\mathrm{mBq} \mathrm{cm}^{-2}$

and in Table 2 for surface contaminations. The latter are calculated by comparing the screening measurement with simulations of ${ }^{40} \mathrm{~K}$ located one monolayer $\left(10^{-10} \mathrm{~m}\right)$ below the surface and ${ }^{210} \mathrm{~Pb}$, which is implanted in the material by the recoil of the decay of ${ }^{222} \mathrm{Rn}$ and its daughters. The typical measurement duration for these screening and background measurements was approximately a month each.

Typical concentration of contamination in pure metals are in the order of $0.1 \mathrm{mBq} \mathrm{kg}^{-1}$ to $1 \mathrm{mBq} \mathrm{kg}^{-1}[24,25]$ and concentrations about $1 \mathrm{~Bq} \mathrm{~kg}^{-1}$ to $2 \mathrm{~Bq} \mathrm{~kg}^{-1}$ in non pure metals and alloys [26], mainly due to the ${ }^{232} \mathrm{Th}$ and ${ }^{238} \mathrm{U}$ chains. So, it is expected that for pure metals like the copper samples, only upper limits can be given. Concentrations of the order of $10^{-4} \mathrm{mBq} \mathrm{cm}^{-2}$ [21] or of $17 \mathrm{mBq} \mathrm{kg}^{-1}$ to $40 \mathrm{mBq} \mathrm{kg}^{-1}$ [27] are expected for ${ }^{210} \mathrm{~Pb}$ in the copper sample and concentrations of the order of $10^{-3} \mathrm{mBq} \mathrm{kg}^{-1}$ for ${ }^{232} \mathrm{Th}$ and ${ }^{238} \mathrm{U}$ [28]. However, this is far below the sensitivity of the used detector. Smaller limits for the copper sample are given by a screening measurement including a similar copper sample with a mass of about $500 \mathrm{~g}$, performed by the team of the Canfranc Underground Laboratory results in upper limits of about $40 \mathrm{mBq} \mathrm{kg}^{-1}$ for the ${ }^{232} \mathrm{Th}$-chain, ${ }^{6}$ about $120 \mathrm{mBq} \mathrm{kg}^{-1}$ for the ${ }^{238} \mathrm{U}_{\text {-chain }}{ }^{7}$ and $25 \mathrm{mBq} \mathrm{kg}^{-1}$ of ${ }^{40} \mathrm{~K}$ [29]. By comparing to the limits resulting from the screening measurement of the copper sample in Tübingen and assuming similar relations between the limits of ${ }^{40} \mathrm{~K}$ surface to bulk contamination and ${ }^{238} \mathrm{U}$ bulk contamination to ${ }^{210} \mathrm{~Pb}$ surface contamination, these limits leads to surface contaminations of less than $25 \mu \mathrm{Bq} \mathrm{cm}^{-2}$ for ${ }^{40} \mathrm{~K}$ and $28 \mu \mathrm{Bq} \mathrm{cm}^{-2}$ for the ${ }^{210} \mathrm{~Pb}$-chain. Similar levels of contamination are also expected for pure aluminum samples [28].

The higher values for plastic (connectors) and the colored circuit board are as well expected. Crude oil and coal are often basic materials for plastics, binders (plastic disper-

\footnotetext{
${ }^{6}$ An equilibrium between the chain members is assumed.
} 
Table 3 The minimum ranges particles have to pass for different volumes

\begin{tabular}{ll}
\hline Volume & Min range \\
\hline MMCs & $100 \mathrm{~nm}$ \\
Thermal baths & $100 \mathrm{~nm}$ \\
$(*)$ Substrate & $1.0 \mu \mathrm{m}$ \\
SQUID chips & $1.0 \mu \mathrm{m}$ \\
Circuit board & $10 \mu \mathrm{m}$ \\
Plugs & $1.0 \mathrm{~mm}$ \\
$(*)$ Copper holder & $1.0 \mathrm{~mm}$ \\
(*)Shielding & $1.0 \mathrm{~mm}$ \\
\hline
\end{tabular}

If a particle has too less energy to travel as far as the minimum range, the particle will not be created and its energy will be deposited locally. Larger volumes, marked with (*), contains sub volumes with smaller minimum range cuts (see Fig. 3)

sions) and solvent. Both, crude oil and coal are known to have relatively large levels of concentrations of natural occurring radionuclides of about tens to hundreds of $\mathrm{Bq} \mathrm{kg}^{-1}$ [30-32]. In newer MMC set-up for low background measurement, polyimide based circuit boards (Kapton) are used due to the very low concentration levels of natural radionuclides [29].

\section{Monte Carlo simulation}

The decays of the radionuclides are simulated using version 10.06.p03 of the GEANT4 toolkit [33]. To study accurately the effect of natural radionuclides located in the surrounding of the MMCs and to decrease computation time as much as possible, the predefined particle production energy thresholds are configured. If a potentially produced particle by a process with infrared divergence, such as bremsstrahlung or $\delta$-ray production, the particle will only be generated, if its kinetic energy is above a given energy threshold. If the kinetic energy of the potentially produced particles is below the energy threshold, their kinetic energy is deposited locally. Particles generated by other processes like the radioactive decay or atomic de-excitation are not affected by this cut.

\subsection{Simulation set-up}

In order to produce as many low energy particles as possible in the close surrounding of the MMCs, the energy threshold is reduced layer by layer. The discussed energy threshold is equivalent to a minimum distance the particles have to pass before getting absorbed. These minimum ranges are shown in Table 3 for different volumes. The larger volumes like the copper holder, aluminum shielding and the substrate of the ECHo-1k chip contain sub volumes as seen in Fig. 3, showing the profile of the set-up around the ECHo-1k chip.

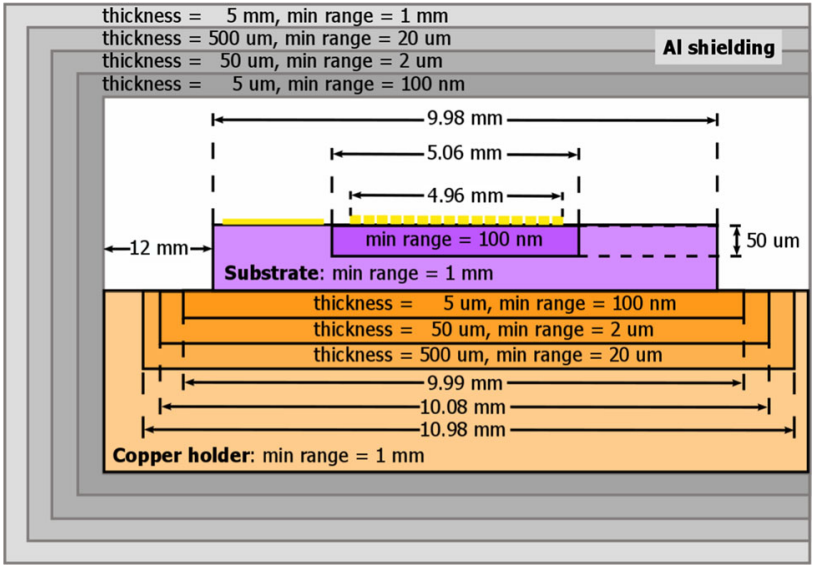

Fig. 3 Non-scaled scheme of the region around the ECHo-1k chip. The minimum ranges particles have to pass before getting absorbed in different layers of the larger volumes and their dimensions are shown. Picture taken from [12]

The simulations are divided into two sets. If the radionuclides are uniformly distributed in the volume materials, they are called bulk-distributed and if the radionuclides are located only on the surface of the volumes, ${ }^{7}$ they are called surfacedistributed. The full decay chains of the nuclides are simulated. The large number of simulated events of the order of $10^{6}$ to $10^{8}$ for each nuclide are needed to obtain high statistics describing the deposited energies of less than $6 \mathrm{keV}$ in the MMC pixels to study the shape of the spectrum in this region. For levels of radioactivity as high as resulting from the screening measurement, the number of simulated decays corresponds to very long measuring times of the order of a few thousand days (compare to Tables 4 and 5). Since no screening measurements are available for the detector-pixels and the aluminum shielding until now, the upper limits of concentrations of the copper sample (from the measurement in the Canfranc Underground Laboratory) is used for the detector pixels, the shielding and copper holder. The two copper samples screened in Tübingen and Canfranc have different shapes. Nevertheless, the same ratio between bulk and surface contamination resulting from the measurement in Tübingen is also assumed for the copper sample used in Canfranc. In order to safe computation time, radionuclides are only placed at the first quarter (next to ECHo-1k chip) of the circuit board. Naturally occurring radionuclides in the connectors are not simulated. For comparison, the simulation of $28 \cdot 10^{6}$ decays of ${ }^{238} \mathrm{U}$ and its daughters in the bulk of the part of the circuit board next to the ECHo-1k chip results in 846 particles hitting the MMCs, while the simulation of $3 \cdot 10^{6}$ decays of ${ }^{238} \mathrm{U}$ and its daughters in the bulk of the rest of the circuit board results only in two hits. Thus, by considering

\footnotetext{
7 The primary nuclides $\left({ }^{40} \mathrm{~K},{ }^{238} \mathrm{U}\right.$ and $\left.{ }^{232} \mathrm{Th}\right)$ are placed $10^{-10} \mathrm{~m}$ below the surface. Due to the recoil energy, daughter nuclides, like ${ }^{210} \mathrm{~Pb}$, can be implanted at larger depths.
} 
Table 4 Number of simulated bulk-distributed decay chains

\begin{tabular}{llll}
\hline & ${ }^{238} \mathrm{U}$-chain & ${ }^{232}$ Th-chain & ${ }^{40} \mathrm{~K}$ \\
\hline Absorber & & & \\
$\quad$ Simulated decays & $1.5 \cdot 10^{6}$ & $1.5 \cdot 10^{6}$ & $1.5 \cdot 10^{6}$ \\
Pixel-days & $3.2 \cdot 10^{11}$ & $6.9 \cdot 10^{11}$ & $1.1 \cdot 10^{11}$ \\
Shielding & & & \\
Simulated decays & $1.2 \cdot 10^{7}$ & $1.2 \cdot 10^{7}$ & $2.4 \cdot 10^{7}$ \\
Days & $6.4 \cdot 10^{4}$ & $1.4 \cdot 10^{5}$ & $4.4 \cdot 10^{4}$ \\
Pixel-days & $4.6 \cdot 10^{6}$ & $1.0 \cdot 10^{7}$ & $3.2 \cdot 10^{6}$ \\
Copper holder & & & \\
Simulated decays & $2.8 \cdot 10^{7}$ & $1.3 \cdot 10^{7}$ & $1.3 \cdot 10^{7}$ \\
Days & $1.5 \cdot 10^{5}$ & $1.5 \cdot 10^{5}$ & $2.3 \cdot 10^{4}$ \\
Pixel-days & $1.1 \cdot 10^{7}$ & $1.1 \cdot 10^{7}$ & $1.7 \cdot 10^{6}$ \\
Circuit board & & & \\
Simulated decays & $2.6 \cdot 10^{6}$ & $2.6 \cdot 10^{6}$ & $1.1 \cdot 10^{7}$ \\
Days & $1.4 \cdot 10^{3}$ & $6.7 \cdot 10^{2}$ & $6.1 \cdot 10^{3}$ \\
Pixel-days & $9.9 \cdot 10^{4}$ & $4.9 \cdot 10^{4}$ & $4.4 \cdot 10^{5}$ \\
\hline
\end{tabular}

The equivalent experiment durations correspond to levels of activities as high as the results/limits of the screening measurement and a set-up including 72 pixels

only events due to radionuclides in the circuit board, it can be assumed, that about $94 \%$ of all particles hitting the detector pixels, are produced by particles created in the part of the circuit board, which is next to the detector pixels. The connectors are relatively far away from the active volume and the aluminum shielding is between the connectors and the active volume (except of the middle connector). They have similar levels of contamination as the circuit board, but result in a smaller solid angle coverage with respect to the active volume. Thus, radionuclides in the connectors should have less influence on the background, by comparing to radionulcides in the circuit board. Radionuclides in the substrate are also not simulated yet, because metallic contamination of less than $10^{11}$ atoms $\mathrm{cm}^{-3}$ can easily be achieved [34,35], which is equal to an activity of less than $10 \mu \mathrm{Bq} \mathrm{kg}^{-1}$.

Following, the values of expected count rates in the last $10 \mathrm{eV}$ below $Q_{\mathrm{EC}}$ are determined by fitting an exponential decay to the spectra in the $1 \mathrm{keV}$ to $5 \mathrm{keV}$ range and averaging of the $2.6 \mathrm{keV}$ to $3 \mathrm{keV}$ range. The obtained value is further scaled with the (upper limit) activity resulting from the screening measurement.

\subsection{Radionuclides in single pixels}

\section{$6.2 .1{ }^{238} U$-chain}

The energy deposition caused by bulk-distributed radionuclides of the ${ }^{238} \mathrm{U}$-chain in the absorbers of the MMCs is shown in Fig. 4. The colors indicate the fractions of the
Table 5 Number of simulated surface-distributed decay chains

\begin{tabular}{lll}
\hline & ${ }^{210} \mathrm{~Pb}-$ chain & ${ }^{40} \mathrm{~K}$ \\
\hline Absorber & & \\
Simulated decays & $1.0 \cdot 10^{6}$ & $1.0 \cdot 10^{6}$ \\
Pixel-days & $1.5 \cdot 10^{10}$ & $1.8 \cdot 10^{9}$ \\
Shielding & & \\
Simulated decays & $2.0 \cdot 10^{6}$ & $2.3 \cdot 10^{7}$ \\
Days & $3.4 \cdot 10^{4}$ & $3.1 \cdot 10^{4}$ \\
Pixel-days & $2.5 \cdot 10^{6}$ & $2.2 \cdot 10^{6}$ \\
Copper holder & & \\
Simulated decays & $3.0 \cdot 10^{6}$ & $6.0 \cdot 10^{7}$ \\
Days & $1.6 \cdot 10^{5}$ & $2.6 \cdot 10^{5}$ \\
Pixel-days & $1.2 \cdot 10^{7}$ & $1.8 \cdot 10^{7}$ \\
Circuit board & & \\
Simulated decays & $2.6 \cdot 10^{6}$ & $6.7 \cdot 10^{6}$ \\
Days & $6.9 \cdot 10^{3}$ & $6.0 \cdot 10^{3}$ \\
Pixel-days & $5.0 \cdot 10^{5}$ & $4.3 \cdot 10^{5}$ \\
\hline
\end{tabular}

The equivalent experiment durations correspond to levels of activities as high as the results/limits of the screening measurement and a set-up including 72 pixels

summed spectra caused by the nuclides of the decay chain. The $\alpha$-decaying isotopes have only a minor effect on the spectrum in the energy region below $6 \mathrm{keV}$ and are not shown, except of ${ }^{238} \mathrm{U}$. However, the emitted $\alpha$-particles can leave the absorbers and hit the material surrounding the MMC array. Thus, structures at $1.5 \mathrm{keV}$ and $1.7 \mathrm{keV}$ can be identified, which corresponds to aluminum and silicon K-shell X-rays and Auger electrons generated in the aluminum shielding and the substrate. In addition, $\delta$-ray produced by $\alpha$-particles can deposit energy in the absorbers. Events caused by $\alpha$-particles, hitting the surrounding, should show coincidences among pixels, meaning that in one pixel energy is deposited by products of the $\alpha$-decay. Another pixel is hit by X-ray, Auger electrons and/or $\delta$-ray generated by the $\alpha$-particle in the surrounding. At higher energies, structures like the $47 \mathrm{keV}^{210} \mathrm{~Pb}$ edge, the peak at $73.9 \mathrm{keV}$ due to the decay of the metastable $\mathrm{J}^{\mathrm{P}}$ state of ${ }^{234} \mathrm{~Pa}$ and exemplary the edge around $72 \mathrm{keV}$ caused by ${ }^{238} \mathrm{U}$, are visible. By assuming an equilibrium of the members of the ${ }^{238} \mathrm{U}$-chain and accumulated activity level of $0.12 \mathrm{~Bq} \mathrm{~kg}^{-1}$, which is equal to about $7 \cdot 10^{8}$ atoms of the ${ }^{238} \mathrm{U}$-chain in each MMC or $10^{-8}$ of the absorber material, a signal count rate of about $(3.8 \pm 0.1) \cdot 10^{-9}$ day $^{-1}$ pixel $^{-1}$ is expected in the ROI.

Figure 5 shows the spectrum caused by surface-distributed ${ }^{238} \mathrm{U}$ on the surface of an absorber. As in the case of bulk-distribution, the summed spectrum has no structures at low energies and the $\alpha$-decaying isotopes have only a minor effect on the spectrum below $6 \mathrm{keV}$. On the other hand, many features can be observed at higher energies. 

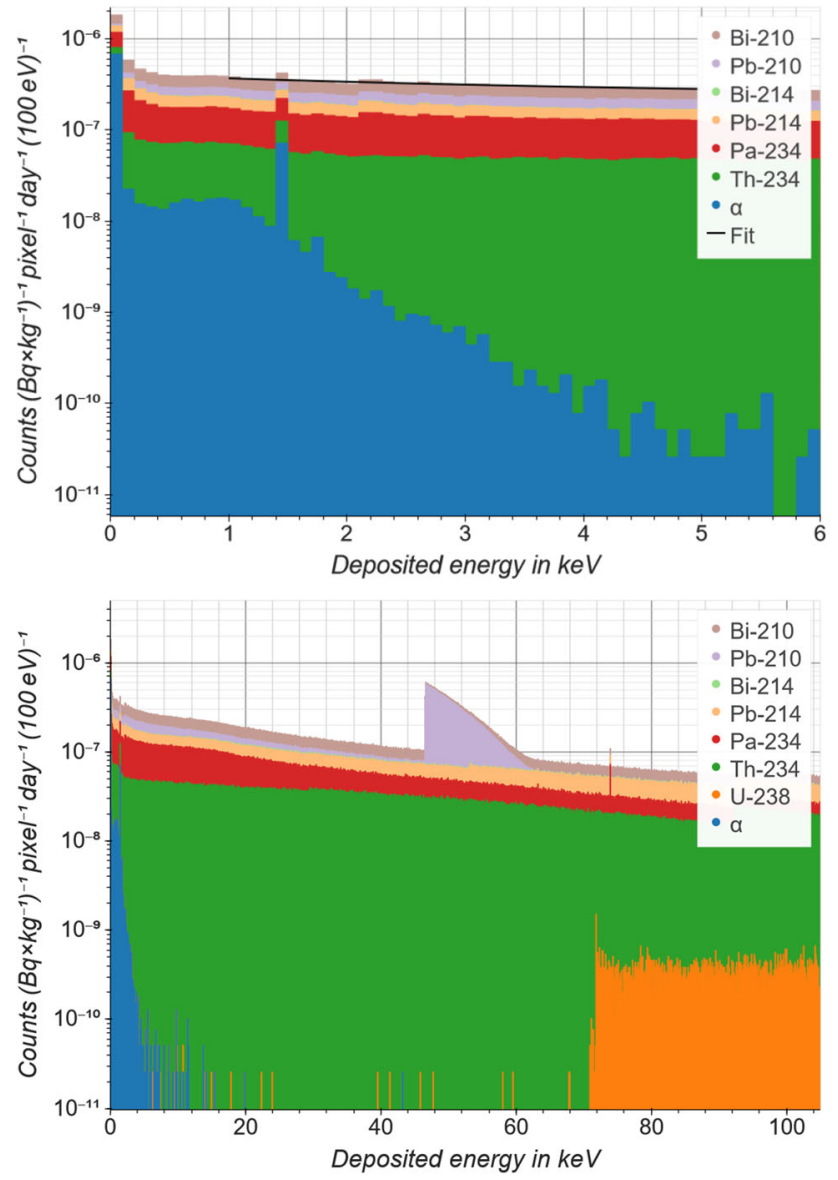

Fig. 4 Simulated spectrum of deposited energies in the MMC absorbers caused by the bulk-distributed ${ }^{238} \mathrm{U}$-chain. The fractions due to the single nuclides of this chain are colored. The summed spectrum is structure-less for low energies. Nuclides decaying by an $\alpha$-decay change the spectrum only minor at low energies, exemplary shown for ${ }^{238}$ U. However, $\alpha$-particles can escape the absorber and hit the aluminum shielding or the substrate. Thus, aluminum K X-ray and Auger electrons and silicon (substrate) K X-ray and Auger electrons as well as $\delta$-ray generated by $\alpha$-particles can hit the absorbers

These occur, because not all decay products deposit their energy in the absorber. The peaks due to the $\alpha$-decaying isotopes are visible at energies of their Q-value reduced by the energy of the emitted $\alpha$ particles, which is the recoil energy of the daughter nucleus. For example ${ }^{238} \mathrm{U}$ : $4270 \mathrm{keV}-4198 \mathrm{keV}=72 \mathrm{keV}$. More than one peak is visible at these energies, because the energy released in deexcitation process of gold atoms (excited during the nuclear recoil) emitted either as Auger electrons or X-rays, which can escape the absorber. For surface-distribution, the $186 \mathrm{keV}$ gamma of ${ }^{226} \mathrm{Ra}$ has a higher path length in the absorbers compared to bulk-distribution and so, has a higher probability to ionize. Thus, the $68 \mathrm{keV} \mathrm{KL} \mathrm{X-ray} \mathrm{line} \mathrm{of} \mathrm{gold} \mathrm{is}$ visible. ${ }^{234} \mathrm{Th}$ provides many features, the $29 \mathrm{keV}, 63 \mathrm{keV}$, $74 \mathrm{keV}$ and $92 \mathrm{keV}$ gamma levels as well as the corresponding X-ray escape peaks. Note that not only gold X-ray can
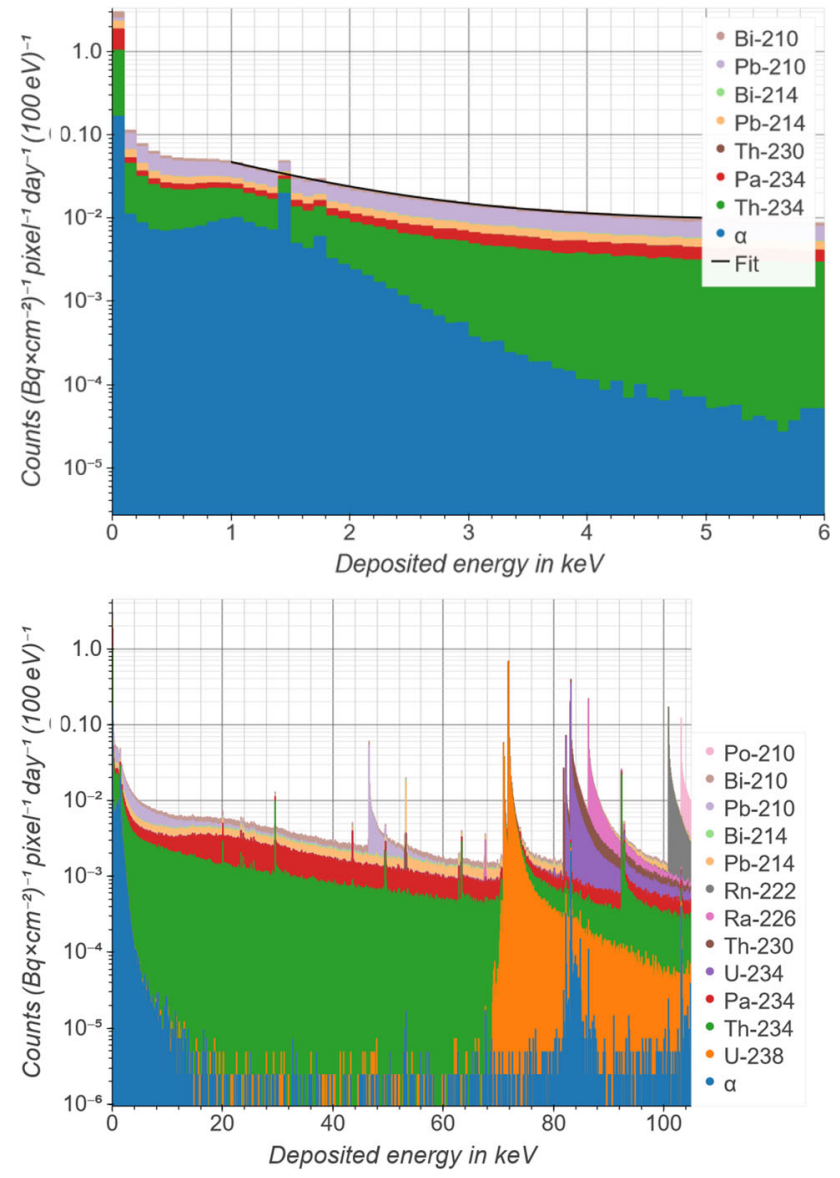

Fig. 5 Simulated spectrum of deposited energies in the MMC absorbers caused by the surface-distributed ${ }^{238} \mathrm{U}$-chain on the absorbers. The fractions due to the single nuclides of this chain are colored. The $\alpha$-particles can be emitted by any isotope. For low energies, the summed spectrum has no structures. Nuclides decaying by an $\alpha$-decay change the spectrum only minor at low energies. At higher energies however, these nuclides provide many features

escape, but also X-ray emitted by the daughter nuclides. From ${ }^{234} \mathrm{~Pa}$, the $43 \mathrm{keV}$ gamma level and from ${ }^{214} \mathrm{~Pb}$, excited during the $\alpha$-decay, the $53 \mathrm{keV}$ level can be identified. Also, at energies above $80 \mathrm{keV}, \alpha$-particles generate more structures. Due to the recoil of the nucleus, the daughter nuclide of an $\alpha$-decaying nuclide can leave the absorber. Thus, there is a chance that only the $\alpha$-particle emitted by these daughters can deposit energy in the absorbers. Only ${ }^{210} \mathrm{~Pb}$ and its daughters ${ }^{210} \mathrm{Bi}$ and ${ }^{210} \mathrm{Po}$ should be surface-distributed due to accumulated ${ }^{222} \mathrm{Rn}^{8}$

With an activity concentration of $0.028 \mathrm{mBq} \mathrm{cm}^{-2}$, a count rate of about $(1.9 \pm 0.1) \cdot 10^{-8} \mathrm{day}^{-1}$ pixel $^{-1}$ is expected in the ROI from these radionuclides, which only corresponds to about 200 atoms on each MMC surface.

$\overline{8{ }^{222} \mathrm{Rn},{ }^{218} \mathrm{Po}},{ }^{214} \mathrm{~Pb},{ }^{214} \mathrm{Bi}$ and ${ }^{214} \mathrm{Po}$ have lifetimes of up to a few days. Thus, these nuclides decay fast, if the material is not exposed to air. 

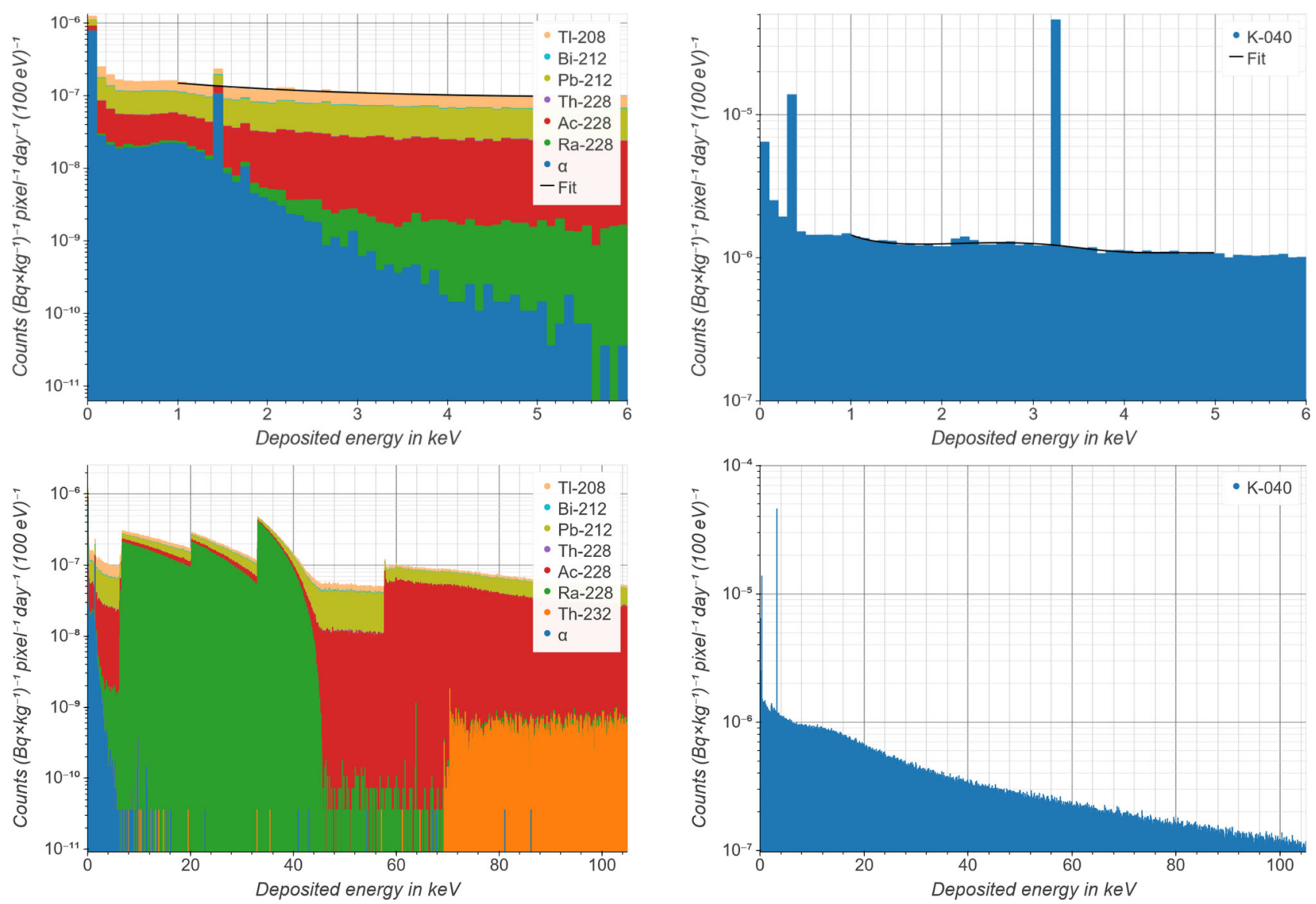

Fig. 6 Simulated spectrum of deposited energies in the MMC absorbers caused by the bulk-distributed ${ }^{232}$ Th-chain as in the absorbers. The fractions due to the single nuclides of this chain are colored. The $\alpha$-particles can be emitted by any isotope. No special structures can be observed for low energies. At higher energies, the ${ }^{228} \mathrm{Ra}$ gamma levels at $6.5 \mathrm{keV}, 20 \mathrm{keV}$ and $33 \mathrm{keV}$ can be identified, as well as the $58 \mathrm{keV}$ level of the decay of ${ }^{228} \mathrm{Ac}$

\subsection{2 ${ }^{232}$ Th-chain}

The effect of bulk-distributed ${ }^{232} \mathrm{Th}$ is shown in Fig. 6. As in the case of bulk-distributed ${ }^{238} \mathrm{U}, \alpha$-decaying isotopes have only a negligible effect on the spectrum at low energies. The summed spectra has no structures in the low energy region, but a few features can be identified at higher energies. The three ${ }^{228} \mathrm{Ra}$ edges at around $6.5 \mathrm{keV}, 20 \mathrm{keV}$ and $33 \mathrm{keV}$ can be seen. Also, the $58 \mathrm{keV}$ excitation level of ${ }^{228} \mathrm{Th}$, the daughter of ${ }^{228} \mathrm{Ac}$ is visible as well as exemplary the edge of ${ }^{232} \mathrm{Th}$. In case of ${ }^{228} \mathrm{Ra}$, there is a chance that ${ }^{228}$ Ac with a half life of about 6 hours decays simultaneously (the time interval used in the simulation to generate pile-up events is $\delta t<1 \mathrm{~ms}$ ) and thus, higher energies than the Q-value of ${ }^{228} \mathrm{Ra}$ of about $46 \mathrm{keV}$ can be measured. For an activity concentration of $0.04 \mathrm{~Bq} \mathrm{~kg}^{-1}$ (about $10^{9}$ atoms per MMC or $10^{-8}$ of the absorber material), a count rate of

Fig. 7 Simulated spectrum of deposited energies in the MMC absorbers caused by the bulk-distributed ${ }^{40} \mathrm{~K}$ in the absorbers. The $\beta$ spectrum is flat for low energies and the Ar shell levels due to the $\mathrm{EC} / \beta^{+}$-decay can be seen

about $(4.5 \pm 0.1) \cdot 10^{-10}$ day $^{-1}$ pixel $^{-1}$ is expected in the ROI.

\section{$6.2 .3{ }^{40} K$}

The resulting simulated spectrum of bulk-distributed ${ }^{40} \mathrm{~K}$ in an absorber can be seen in Fig. 7. The only visible features are the $\mathrm{Ar}$ shell levels due to the $\mathrm{EC} / \beta^{+}$-decay at $0.3 \mathrm{keV}$ and $3.2 \mathrm{keV}$. The $\beta$-spectrum is flat for energies below $6 \mathrm{keV}$.

For an activity concentration of $0.025 \mathrm{~Bq} \mathrm{~kg}^{-1}$ (about $10^{8}$ atoms per MMC or $7 \cdot 10^{-9}$ of the absorber material), a count rate of about $(3.2 \pm 0.1) \cdot 10^{-9} \mathrm{day}^{-1}$ pixel $^{-1}$ is expected in the last $10 \mathrm{eV}$ below $Q_{\mathrm{EC}}$. Figure 8 shows the spectrum caused by surface-distributed ${ }^{40} \mathrm{~K}$ on the surface of an absorber. Compared to bulk-distributed ${ }^{40} \mathrm{~K}$, the lines due to the $\mathrm{EC} / \beta^{+}$. decay are broader. This happens, because not all Auger electrons and X-ray deposit all their energy in the absorber. For an activity concentration of $0.025 \mathrm{mBq} \mathrm{cm}^{-2}$ (about $3 \cdot 10^{10}$ atoms per MMC-surface or $0.5 \%$ of the surface atoms), a 

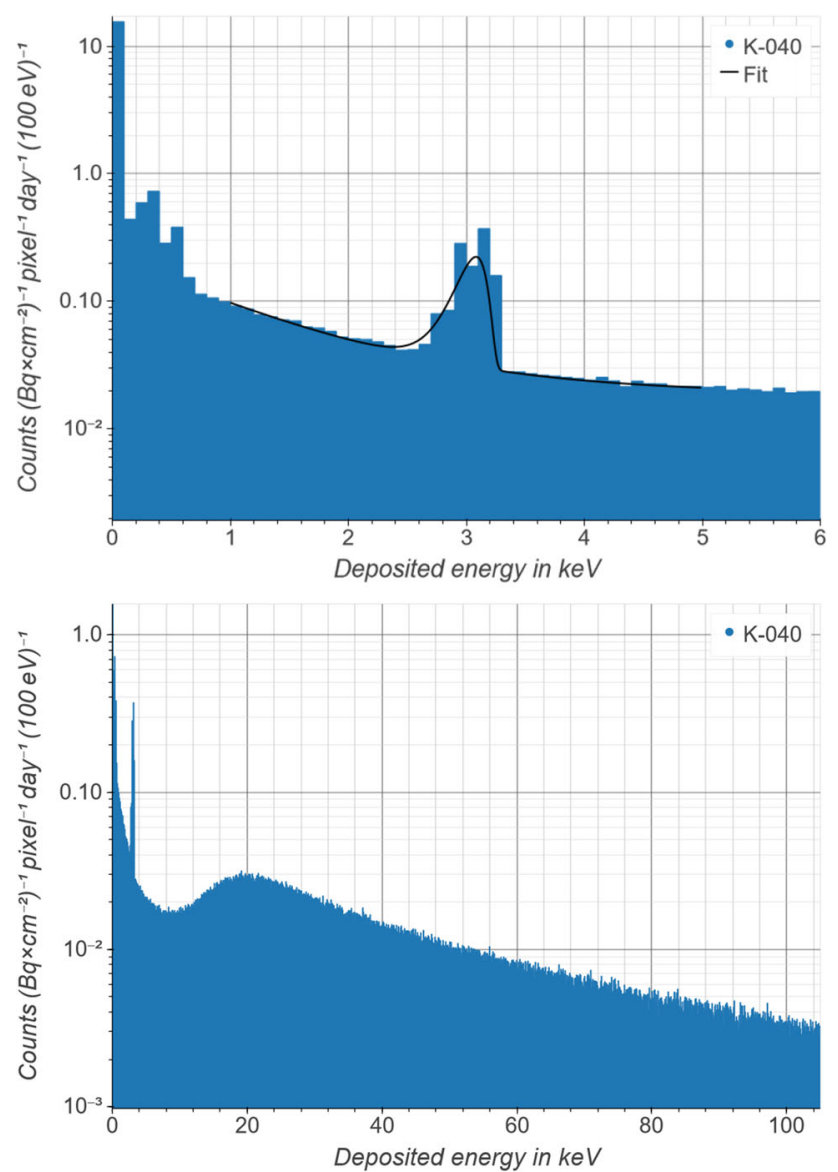

Fig. 8 Simulated spectrum of deposited energies in the MMC absorbers caused by the surface-distributed ${ }^{40} \mathrm{~K}$ on the absorbers. The Ar lines are visible. But, in comparison to bulk contamination, they are broader, because not all X-ray and Auger electrons have to be emitted to the absorber

count rate of about $(2 \pm 1) \cdot 10^{-7}$ day $^{-1}$ pixel $^{-1}$ is expected in the ROI.

\subsection{Radionuclides in the aluminum shielding}

\section{$6.3 .1{ }^{238} U$-chain}

The resulting simulated spectra for bulk- and surfacedistributed ${ }^{238} \mathrm{U}$ in the aluminum shielding can be seen in Figs. 9 and 10 respectively. In case of bulk-distribution, mainly higher energy electrons/ $\beta$-particles deposit energy in the absorbers and thus, mainly the $\beta$-decaying isotopes with higher Q-values $(\geq 1 \mathrm{MeV})$ are listed, but also the excited daughters of ${ }^{214} \mathrm{Po}$ can often emit high energy conversion electrons. The $\alpha$-particles can generate much $\delta$-radiation, which can deposit energy in the absorbers. Mainly the Landau distribution [36] caused by these electrons can be seen. For comparison, the Landau distribution caused by $500 \mathrm{keV}$ electrons passing the absorbers is plotted. Similar to the surfacedistribution of ${ }^{238} \mathrm{U}$ on the absorbers, the spectrum resulting

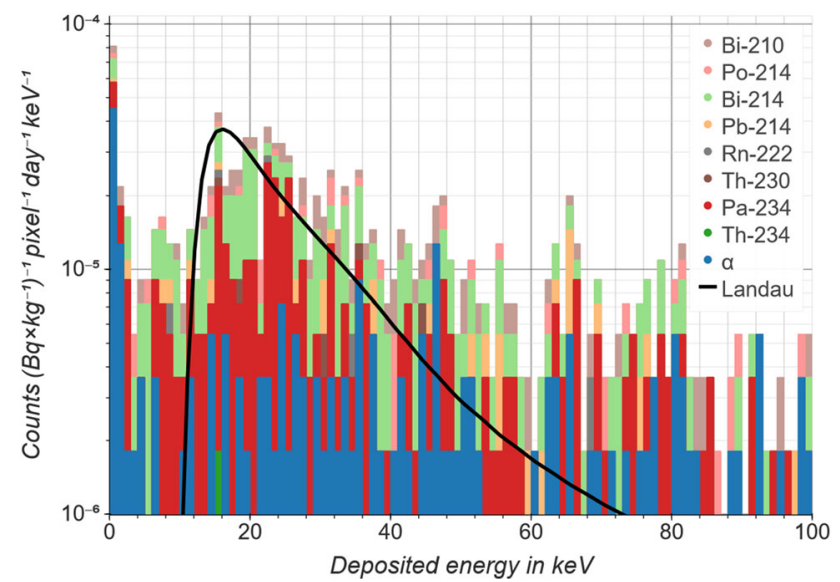

Fig. 9 Simulated spectrum of deposited energies in the MMC absorbers caused by the bulk-distributed ${ }^{238} \mathrm{U}$-chain in the aluminum shielding. No special structures are observable. Mainly, electrons ( $\beta$ particles) deposit energy in the absorbers. A Landau distribution [36] is expected to be caused by these electrons and can be compared to a Landau distribution for $500 \mathrm{keV}$ electrons passing the absorbers [37]

from surface-distributed ${ }^{238} \mathrm{U}$ in the shielding shows peaks from $\alpha$-decaying isotopes, which can be seen at energies at the $\mathrm{Q}$-values minus the energy of the emitted $\alpha$-particle. Also, the $\alpha$-particles can produce $\delta$-radiation and X-ray, which results in deposited energies lower than $6 \mathrm{keV}$. An activity concentration of bulk-distributed ${ }^{238} \mathrm{U}$ of $0.12 \mathrm{~Bq} \mathrm{~kg}^{-1}$ results in a count rate of less than $2.7 \cdot 10^{-8} \mathrm{day}^{-1}$ pixel $^{-1}$ at $90 \%$ C.L. in the ROI and surface-distributed ${ }^{210} \mathrm{~Pb}$ and daughters with a level of $0.028 \mathrm{mBq} \mathrm{cm}^{-2}$ result in a count rate of about $(6 \pm 1) \cdot 10^{-8}$ day $^{-1}$ pixel $^{-1}$ in the ROI.

\subsection{2 ${ }^{232}$ Th-chain}

${ }^{232} \mathrm{Th}$ in and on the aluminum shielding results in the same structures like the ones resulting from ${ }^{238} \mathrm{U}$ in the shielding, the Landau distribution caused by electrons. Bulk-distributed ${ }^{232} \mathrm{Th}$ with a level of $0.04 \mathrm{~Bq} \mathrm{~kg}^{-1}$ results in a count rate of $(6 \pm 2) \cdot 10^{-9}$ day $^{-1}$ pixel $^{-1}$ in the ROI.

\subsection{3 ${ }^{40} K$}

The spectra resulting from ${ }^{40} \mathrm{~K}$ show no structures. Bulkdistributed ${ }^{40} \mathrm{~K}$ with a level of $0.025 \mathrm{~Bq} \mathrm{~kg}^{-1}$ results in a count rate of $(8 \pm 3) \cdot 10^{-9}$ day $^{-1}$ pixel $^{-1}$ in the ROI and results surface-distributed and with a level of $0.025 \mathrm{mBq} \mathrm{cm}^{-2}$ in a count rate of about $(4 \pm 2) \cdot 10^{-7} \mathrm{day}^{-1}$ pixel $^{-1}$ in the ROI.

\subsection{Radionuclides in the copper holder}

Naturally occurring radionuclides located in the copper holder results in similar spectra as resulting from radionu- 

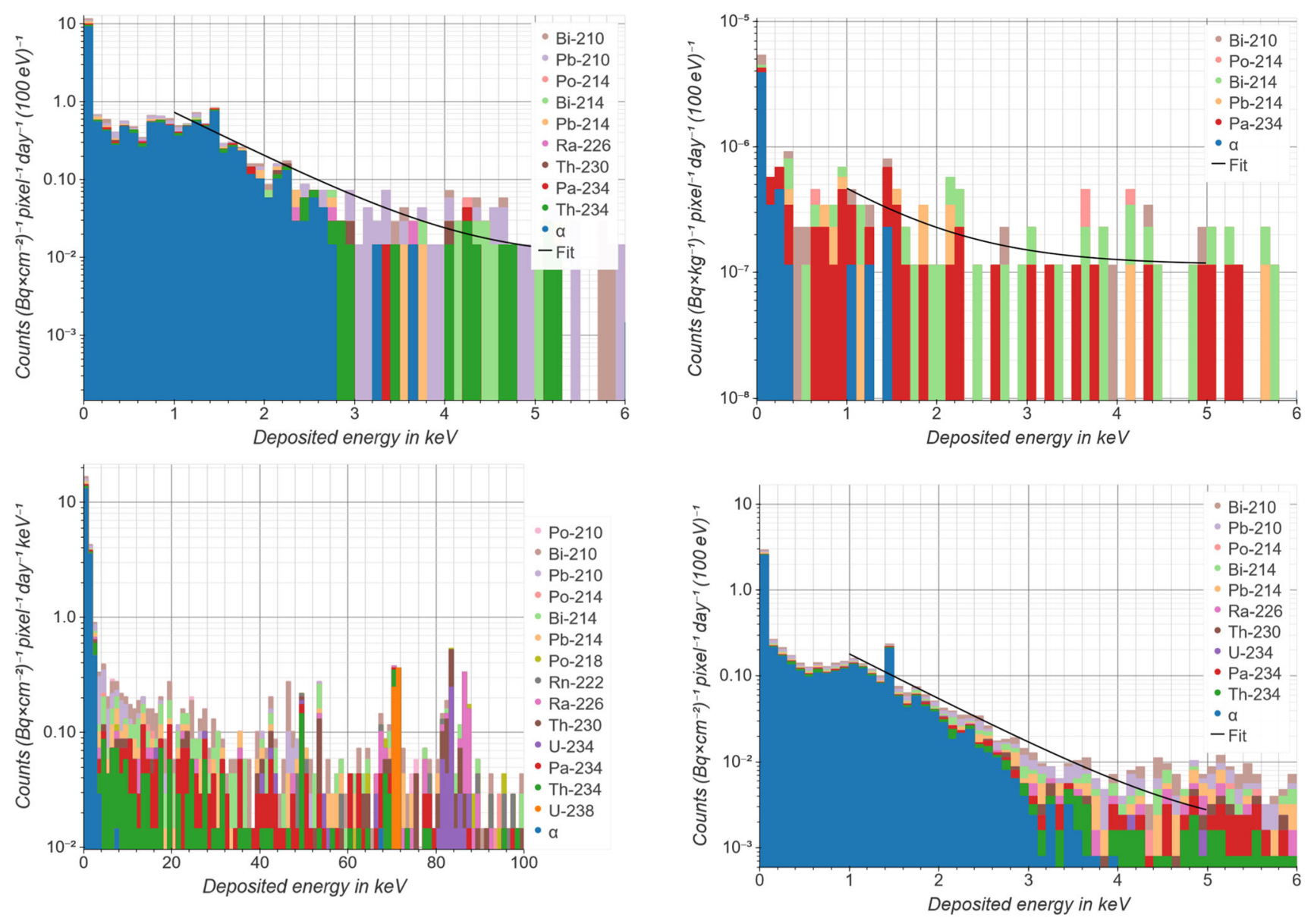

Fig. 10 Simulated spectrum of deposited energies in the MMC absorbers caused by the surface-distributed ${ }^{238} \mathrm{U}$ on the aluminum shielding. Lines caused by the $\alpha$-decaying isotopes can be identified. Also, low energy particles generated by $\alpha$-particles in the surrounding of the MMCs can hit the pixels

clides in the aluminum shielding, but with lower intensities. With the same concentrations as used for the absorbers and shielding, nuclides of the ${ }^{238} \mathrm{U}$-chain cause a count rate of $(6 \pm 1) \cdot 10^{-9}$ day $^{-1}$ pixel $^{-1}$ in the ROI for bulkdistributed nuclides and $(6 \pm 2) \cdot 10^{-9}$ day $^{-1}$ pixel $^{-1}$ for surface-distributed ${ }^{210} \mathrm{~Pb}$. For bulk-distributed ${ }^{232} \mathrm{Th}$, a count rate of about $(2 \pm 1) \cdot 10^{-9} \mathrm{day}^{-1}$ pixel $^{-1}$ in the ROI is expected. Bulk-distributed ${ }^{40} \mathrm{~K}$ results in a count rate of less than $8 \cdot 10^{-9}$ counts day ${ }^{-1}$ pixel $^{-1}$ in the ROI at $90 \%$ C.L. and in about $(1.7 \pm 0.3) \cdot 10^{-8} \mathrm{day}^{-1}$ pixel $^{-1}$, if surfacedistributed.

\subsection{Radionuclides in the circuit board}

Bulk-distributed ${ }^{238} \mathrm{U}$, with a level of $88 \mathrm{~Bq} \mathrm{~kg}^{-1}$ in the circuit board, results in a count rate of about $(1.4 \pm$ $0.4) \cdot 10^{-6} \mathrm{day}^{-1} \mathrm{pixel}^{-1}$ in the ROI and in $(1.6 \pm 0.3)$. $10^{-6} \mathrm{day}^{-1} \mathrm{pixel}^{-1}$ for the surface-distributed ${ }^{210} \mathrm{~Pb}$-chain with a level of $2.51 \mathrm{mBq} \mathrm{cm}^{-2}$. The spectra of energy

Fig. 11 Simulated spectrum of deposited energies in the MMC absorbers caused by a bulk-distributed ${ }^{238} \mathrm{U}$ and $\mathbf{b}$ surface-distributed ${ }^{238} \mathrm{U}$ in the circuit bard

deposited in the MMCs caused by the ${ }^{238} \mathrm{U}$-chain in the circuit board is shown in Fig. 11 and are similar to the ones caused by ${ }^{238} \mathrm{U}$ and its daughters in the aluminum shielding. For bulk-distributed ${ }^{232} \mathrm{Th}$ with a level of $128 \mathrm{~Bq} \mathrm{~kg}^{-1}$, a count rate of about $(2 \pm 1) \cdot 10^{-6} \mathrm{day}^{-1}$ pixel $^{-1}$ is expected in the ROI. Bulk-distributed ${ }^{40} \mathrm{~K}$ with a level of $6.2 \mathrm{~Bq} \mathrm{~kg}^{-1}$ results in about $(3 \pm 1) \cdot 10^{-7}$ day $^{-1}$ pixel $^{-1}$ in the ROI and in about $(5 \pm 2) \cdot 10^{-7} \mathrm{day}^{-1}$ pixel $^{-1}$, if surface-distributed with a level of $0.53 \mathrm{mBq} \mathrm{cm}^{-2}$.

\subsection{Upper limits}

Upper limits of tolerable concentrations of naturally occurring radionuclides are obtained by fitting an exponential distribution to the spectra and scaled, so that the count rate in the last $10 \mathrm{eV}$ below the Q-value of the ${ }^{163} \mathrm{Ho}$ $\mathrm{EC}$ is equal to the expected rate of unresolved pile-up of about $10^{-6} \mathrm{day}^{-1} \mathrm{pixel}^{-1}$. The resulting concentration limits for bulk- and surface-distributed radionuclides are listed in Tables 6 and 7. 
Table 6 Necessary activity concentrations in $\mathrm{Bq} \mathrm{kg}^{-1}$ for a count rate due to radionuclides equal to the unresolved pile-up count rate in the ROI

\begin{tabular}{lll}
\hline Volume & Contamination & Upper limit \\
\hline Absorber & ${ }^{238} \mathrm{U}$-chain & $32 \pm 1$ \\
Absorber & ${ }^{232} \mathrm{Th}$-chain & $89 \pm 2$ \\
Absorber & ${ }^{40} \mathrm{~K}$ & $7.9 \pm 0.3$ \\
Shielding & ${ }^{238} \mathrm{U}$-chain & $9 \pm 5$ \\
Shielding & ${ }^{232} \mathrm{Th}$-chain & $7 \pm 3$ \\
Shielding & ${ }^{40} \mathrm{~K}$ & $3 \pm 1$ \\
Copper holder & ${ }^{238} \mathrm{U}$-chain & $20 \pm 4$ \\
Copper holder & ${ }^{232} \mathrm{Th}$-chain & $30 \pm 10$ \\
Copper holder & ${ }^{40} \mathrm{~K}$ & $<29$ at $90 \%$ C.L. \\
PCB & ${ }^{238} \mathrm{U}$-chain & $60 \pm 20$ \\
PCB & ${ }^{232} \mathrm{Th}$-chain & $70 \pm 30$ \\
PCB & ${ }^{40} \mathrm{~K}$ & $22 \pm 6$
\end{tabular}

Table 7 Necessary activity concentration in $\mathrm{mBq} \mathrm{cm}^{-2}$ for a count rate due to radionuclides equal to the unresolved pile-up count rate in the ROI

\begin{tabular}{lll}
\hline Volume & Contamination & Upper limit \\
\hline Absorber & ${ }^{210} \mathrm{~Pb}$-chain & $1.5 \pm 0.1$ \\
Absorber & ${ }^{40} \mathrm{~K}$ & $0.11 \pm 0.05$ \\
Shielding & ${ }^{210} \mathrm{~Pb}$-chain & $0.5 \pm 0.1$ \\
Shielding & ${ }^{40} \mathrm{~K}$ & $0.06 \pm 0.02$ \\
Copper holder & ${ }^{210} \mathrm{~Pb}$-chain & $5 \pm 2$ \\
Copper holder & ${ }^{40} \mathrm{~K}$ & $1.5 \pm 0.3$ \\
PCB & ${ }^{210} \mathrm{~Pb}$-chain & $1.5 \pm 0.3$ \\
PCB & ${ }^{40} \mathrm{~K}$ & $1.1 \pm 0.4$ \\
\hline
\end{tabular}

Compared to the results of the screening measurement of Chapter 5, the upper limits of tolerable concentrations are much larger than the upper limits determined in the screening campaign, except of the limits for the circuit board. By assuming that ${ }^{40} \mathrm{~K}$ is only surface-distributed, a total count rate of $(6 \pm 1) \cdot 10^{-6}$ day $^{-1}$ pixel $^{-1}$ is expected in the ROI, mainly due to radionuclides in the circuit board (about $87 \%$ ), which is in the same order than the unresolved pile-up. The expected background spectrum is shown in Fig. 12. In future stages of the ECHo experiment, the circuit board will be made of polyimide-based materials, resulting in lower concentrations of radionuclides. However, the background level of the order of $10^{-6} \mathrm{day}^{-1}$ pixel $^{-1}$ does not danger the ECHo- $1 \mathrm{k}$ phase, since this background results in a count rate of about $10^{-5}$ day $^{-1}$ pixel $^{-1}$ in the energy range of $Q_{\mathrm{EC}} \pm 100 \mathrm{eV}$ and thus, only one background event is expected with an exposure of five month and 64 pixels.

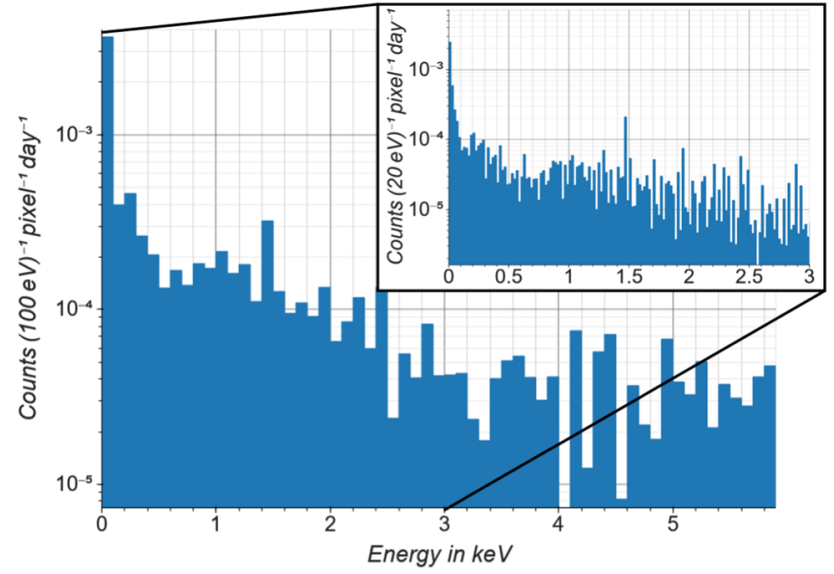

Fig. 12 Total expected spectrum of deposited energies caused by natural occurring radionuclides in materials next to the detector arrays. The only visible structure is caused by $1.486 \mathrm{keV}$ aluminum X-rays

\section{Background measurement}

Due do the double-meander pick-up coil geometry of the MMCs, two pixels are readout by the same SQUID. In the following, these pairs of pixels are called channels. The ECHo$1 \mathrm{k}$ high statistics spectrum has been acquired in two independent runs between Dec 2019 and May 2020. In both runs, several channels of two ECHo-1k chips have been used. A description of the detectors used for this ECHo-1k measurement can be found in [9]. Here, we describe the analysis of events acquired in five asymmetric channels, meaning channels in which only one pixel was implanted with ${ }^{163}$ Ho. The pixels without ${ }^{163}$ Ho can therefore be used for background measurements. In total, about 241 pixel-days of data of pure background have been analyzed.

The thermodynamical properties of the two pixels of one channel are nearly identical. In order to separate events caused by particles, which deposit their complete energies in the pixels from events caused by particles, which are not fully stopped in the pixels, signals due to the decay of ${ }^{163} \mathrm{Ho}$ of the implanted pixels are analyzed first. The signal pulse shape can be described by several parameters and are calibrated by using ${ }^{163}$ Ho events, which correspond to events due to particles fully stopped in the pixels. This calibration, as well as the energy calibration, are adapted for the analysis of events in the background pixels.

The pulse shape analysis is based on the analysis developed to recognize muon induced events [12,38] and modified to select ${ }^{163}$ Ho-like events. Five parameters are obtained from each pulse, its integral, rise time and amplitude on the one hand, and on the other hand, the pulse is compared to a template and is correlated with the same template. Events, caused by particles depositing their complete energy in the pixels, are identified by comparing these parameters to each other. 


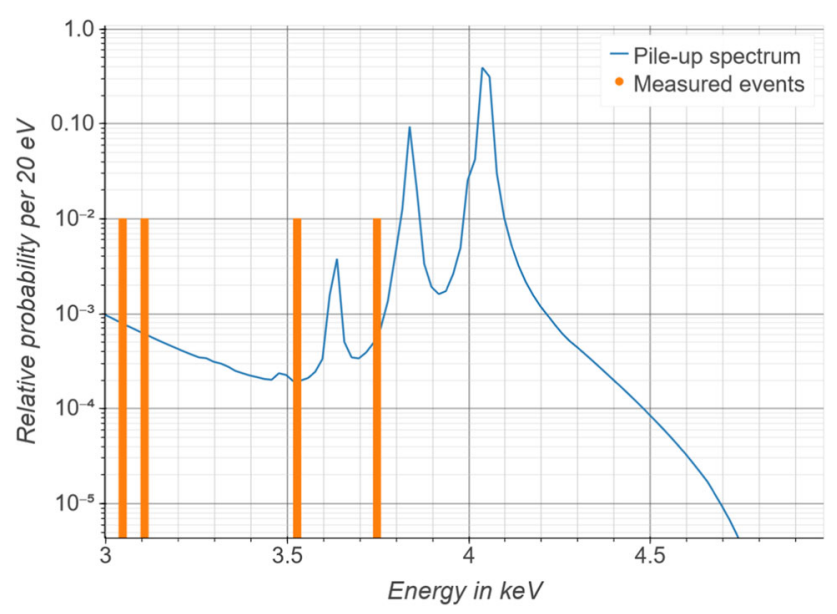

Fig. 13 Normalized energy spectra of unresolved pile-up and measured events in 241 pixel-days

\subsection{Background estimation}

Events with energies above $3 \mathrm{keV}$ are chosen from the data set containing data of the five ${ }^{163} \mathrm{Ho}$-loaded pixels. Above $3 \mathrm{keV}$, only unresolved pile-up events and background events are expected, while above $6 \mathrm{keV}$ only background events are expected. However, due to ADC settings the pulse height is limited to about $5 \mathrm{keV}$. We measured four events with energies between $3 \mathrm{keV}$ and $5 \mathrm{keV}$ in 241 pixel-days after pulse shape analysis. In the same energy region, we expect about 0.7 counts due to unresolved pile-up events. However, by considering the pile-up spectrum, the probability that one count of these events is caused by pile-up is about $22 \%$ and less then $0.2 \%$ that more then one of these counts are caused by pile-up. The expected pile-up spectrum normalized to the $3 \mathrm{keV}$ to $5 \mathrm{keV}$ energy region and the measured events are shown in Fig. 13. Thus, these events are more likely caused by a different background source. Assuming a flat background level, this results in a background level of $(8 \pm 4) \cdot 10^{-5}$ pixel $^{-1}$ day $^{-1}$ in the last $10 \mathrm{eV}$ before $Q_{\mathrm{EC}}$, which is higher than the estimated background due to radionuclides of $6 \cdot 10^{-6}$ pixel $^{-1}$ day $^{-1}$.

The energy spectrum recorded by the background pixels, which are not loaded with ${ }^{163} \mathrm{Ho}$, is shown in Fig. 14. After the pulse shape analysis, no events above $3 \mathrm{keV}$ are measured. This looks like a contradiction to the observation from the ${ }^{163} \mathrm{Ho}$-loaded pixels. However, the $95 \%$ confidence intervals for the mean of the Poisson distribution are $[0,3.69)$ for no observations and $[1.09,10.24)$ for four counts. Accordingly, there is an overlap of the sets. ${ }^{166 m} \mathrm{Ho}$ can be excluded as background source, since a fraction of ${ }^{166 m} \mathrm{Ho} /{ }^{163} \mathrm{Ho}>10^{-5}$ would be needed to explain these events [8] and is more than four orders of magnitudes higher than achieved. A fraction of $10^{-4}$ can be achieved due to chemical purification [15] and a further reduction of five orders of magnitude by mass sepa-

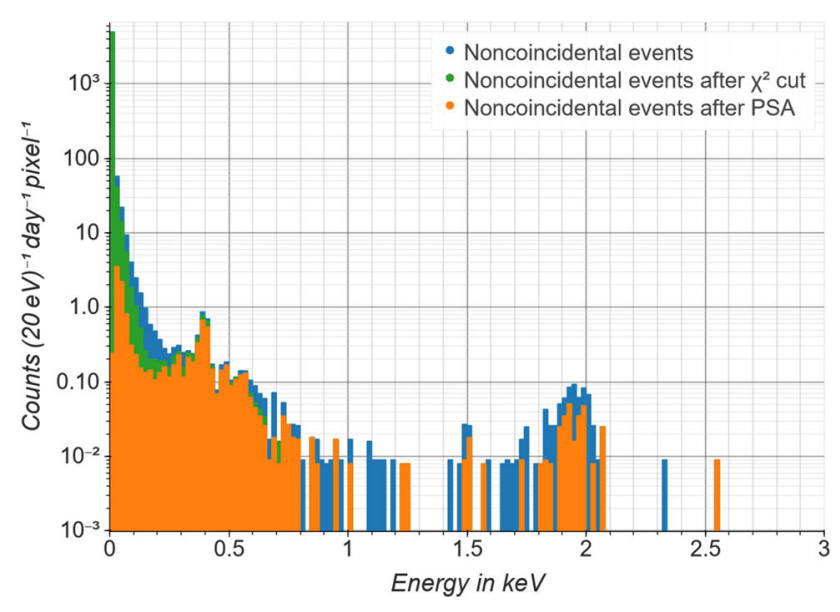

Fig. 14 Energy distribution of non-coincidental events measured by the background pixels. The blue spectrum shows all measured events. The orange spectrum shows the actual background spectrum

ration [16], resulting in a fraction of ${ }^{166 m} \mathrm{Ho} /{ }^{163} \mathrm{Ho}<10^{-9}$. Measurements over a larger energy range are planned within the coming ECHo runs in order to be sensitive to structures at higher energies, which could be used to improve the understanding of the background sources. A ${ }^{163} \mathrm{Ho}$-like background can be observed in the spectrum recorded by the background pixels. This spectrum can be observed for each pixel and can neither be described by any simulations of natural radionuclides described in Sect. 6 so far or by muon caused events [12]. The measured spectrum could correspond to an activity of about $30 \mu \mathrm{Bq}$ of ${ }^{163} \mathrm{Ho}$ per pixel, which corresponds to about $6 \cdot 10^{6}$ atoms per pixel. The observed lines, which could represent the $\mathrm{M}$ lines around $2 \mathrm{keV}$ and $\mathrm{N}$ lines round $400 \mathrm{eV}$ of the ${ }^{163} \mathrm{Ho} \mathrm{EC}$, are slightly shifted to lower energies compared to the ${ }^{163} \mathrm{Ho}$ EC spectrum. Also, the fraction of number of events of the $\mathrm{N}$ line to the number of events of the $\mathrm{M}$ line of $5 \pm 1$ is larger than expected from theory with 2.3. The energy shift of the lines and the higher N/M-ratio could be explained by ${ }^{163} \mathrm{Ho}$ located at the surface of the background pixels, because multiple particles could be emitted by the decay. Further, if this is the case, a surface contamination of about $93 \mathrm{mBq} \mathrm{cm}^{-2}$ can be assumed for the whole detector chip. The endpoint region should not be affected by surface-distributed ${ }^{163} \mathrm{Ho}$, because the ${ }^{163} \mathrm{Ho}$ activity in the pixels is five orders of magnitude higher than the potential surface contamination. If the source of this spectrum has different origins, a background level of about $8 \cdot 10^{-5}$ pixel $^{-1}$ day $^{-1}$ can be assumed, since one event was measured between $2.5 \mathrm{keV}$ and $3 \mathrm{keV}$ in 241 pixel-days. In addition, the $1.49 \mathrm{keV}$ line of aluminum X-rays could be observable. 


\section{Summary}

Different natural radionuclides were simulated in different parts of the ECHo set-up. The background in the ROI of the ECHo experiment is aimed to be dominated the unresolved pile-up of about $10^{-6} \mathrm{day}^{-1} \mathrm{pixel}^{-1}$ in the ROI for the ECHo-1k phase with a ${ }^{163} \mathrm{Ho}$-activity per pixel of $1 \mathrm{~Bq}$ and a time resolution of $500 \mathrm{~ns}$. The simulations show that concentrations of radionuclides of the order of $10 \mathrm{~Bq} \mathrm{~kg}^{-1}$ or a few hundreds of $\mu \mathrm{Bq} \mathrm{cm}^{-2}$ are needed to result in signal count rates as high as the signal due to the unresolved pile-up. Screening measurements were performed and concentration levels are given for connectors (which are not used in the simulations) and circuit boards. For copper, only upper limits are given, pointing out that the material is quite pure. The same can be expected for aluminum. In total, a count rate of about $(6 \pm 1) \cdot 10^{-6} \mathrm{day}^{-1} \mathrm{pixel}^{-1}$ is expected in the ROI due to naturally occurring radionuclides in materials in the next surrounding of the detector arrays. This value is higher than the rate of unresolved pile-up, but low enough for the ECHo-1k phase with an exposure of about less than one year. For the future stage of the ECHo experiment, ECHo$100 \mathrm{k}$, different materials (Kapton) are foreseen as material for circuit boards. This should reduce the background due to natural radionuclides of about $90 \%$. Further, a ${ }^{163}$ Ho activity of a few Bq per pixel is foreseen, which increases the signal count rate as well as the pile-up.

Background measurements including ${ }^{163}$ Ho-loaded and -unloaded pixels were analyzed using pulse shape discrimination methods. Only events above the Q-value of the ${ }^{163} \mathrm{Ho}$ EC are considered for data of ${ }^{163}$ Ho-loaded pixels, which can either be caused by unresolved pile-up events or by other sources. We measured a background level of about $8 \cdot 10^{-5}$ day $^{-1}$ pixel $^{-1}$ in the ROI. This count rate is higher compared to the pile-up limit and the expected background due to the simulated radionuclides and muons. On the other hand, the background pixels did not measure any events in the same energy region of above $3 \mathrm{keV}$. The spectrum measured by these background pixels could be caused by surfacedistributed ${ }^{163}$ Ho with about $30 \mu \mathrm{Bq}$ per pixel, from which a count rate of less than $10^{-8}$ day $^{-1}$ pixel $^{-1}$ is expected in the ROI.

Acknowledgements The experiment described in this paper was possible thanks to the DFG Research Unit FOR 2202 Neutrino Mass Determination by Electron Capture in $163 \mathrm{Ho}$, ECHo (funding under JO 451/1-1, GA 2219/2-1). We acknowledge the support of the clean room team of the Kirchhoff-Institute for Physics, Heidelberg University. S. Scholl has been specially acknowledged for providing the material screening measurements.

Data Availability Statement This manuscript has no associated data or the data will not be deposited. [Authors' comment: The data sets used and generated by the analysis are available from the corresponding author on request.]
Open Access This article is licensed under a Creative Commons Attribution 4.0 International License, which permits use, sharing, adaptation, distribution and reproduction in any medium or format, as long as you give appropriate credit to the original author(s) and the source, provide a link to the Creative Commons licence, and indicate if changes were made. The images or other third party material in this article are included in the article's Creative Commons licence, unless indicated otherwise in a credit line to the material. If material is not included in the article's Creative Commons licence and your intended use is not permitted by statutory regulation or exceeds the permitted use, you will need to obtain permission directly from the copyright holder. To view a copy of this licence, visit http://creativecomm ons.org/licenses/by/4.0/.

Funded by SCOAP 3 .

\section{References}

1. L. Gastaldo et al., Eur. Phys. J. Spec. Top. 226(8), 1623 (2017). https://doi.org/10.1140/epjst/e2017-70071-y

2. S. Eliseev et al., Phys. Rev. Lett. 115, 062501 (2015). https://doi. org/10.1103/PhysRevLett.115.062501

3. M. Bra $\beta$, M.W. Haverkort, New J. Phys. 22(9), 093018 (2020). https://doi.org/10.1088/1367-2630/abac72

4. M. Braß et al., Phys. Rev. C 97, 054620 (2018). https://doi.org/10. 1103/PhysRevC.97.054620

5. S. Kempf et al., J. Low Temp. Phys. 193(3), 365 (2018). https:// doi.org/10.1007/s10909-018-1891-6

6. A. Fleischmann et al., AIP Conf. Proc. 1185(1), 571 (2009). https:// doi.org/10.1063/1.3292407

7. S. Scholl, A. Zschocke, J. Jochum, J. Phys. Conf. Ser. 888, 012225 (2017). https://doi.org/10.1088/1742-6596/888/1/012225

8. S. Scholl, J. Phys. Conf. Ser. 718, 062051 (2016). https://doi.org/ 10.1088/1742-6596/718/6/062051

9. F. Mantegazzini et al., Nucl. Instrum. Methods Phys. Res. Sect. A Accel. Spectrom. Detect. Assoc. Equip. (will be submitted). arXiv:2111.09945

10. F. Mantegazzini et al., J. Instrum. 16(08), P08003 (2021). https:// doi.org/10.1088/1748-0221/16/08/p08003

11. R. Hammann et al., Eur. Phys. J. C 81, 963 (2021). https://doi.org/ 10.1140/epjc/s10052-021-09763-9

12. A. Göggelmann et al., Eur. Phys. J. C 81, 363 (2021). https://doi. org/10.1140/epjc/s10052-021-09148-y

13. L. Baudis et al., Eur. Phys. J. C 75, 485 (2015). https://doi.org/10. 1140/epjc/s10052-015-3711-3

14. J. Engle et al., Nucl. Instrum. Methods Phys. Res. Sect. B Beam Interact. Mater. Atoms 311, 131 (2013). https://doi.org/10.1016/j. nimb.2013.06.017

15. H. Dorrer et al., Radiochim. Acta 106(7), 535 (2018). https://doi. org/10.1515/ract-2017-2877

16. T. Kieck et al., Nucl. Instrum. Methods Phys. Res. Sect. A Accel. Spectrom. Detect. Assoc. Equip. 945, 162602 (2019). https://doi. org/10.1016/j.nima.2019.162602

17. S. Chu, L. Ekström, R. Firestone (1999). http://nucleardata.nuclear. lu.se/toi/

18. I.N.D. Services (2019). https://www-nds.iaea.org/relnsd/ vcharthtml/VChartHTML.html/

19. S. Taylor, Geochim. Cosmochim. Acta 28(8), 1273 (1964). https:// doi.org/10.1016/0016-7037(64)90129-2

20. National Research Council (US) Committee on Health Risks of Exposure to Radon (BEIR VI), Helth Effects of Exposure to Radon (National Academics Press (US), Washington (DC), 1999). https://doi.org/10.17226/5499. https://www.ncbi.nlm.nih. gov/books/NBK233262/ 
21. R. Bunker et al., Nucl. Instrum. Methods Phys. Res. Sect. A Accel. Spectrom. Detect. Assoc. Equip. 967, 163870 (2020). https://doi. org/10.1016/j.nima.2020.163870

22. C. Sailer, Realisierung eines Messplatzes für Tieftemperatur Detektoren. Diploma thesis, U. Tubingen (2008)

23. A. Hegai, Gammaspektroskopie mit Germaniumdetektoren. Diploma thesis, U. Tubingen (2010)

24. D. Budjas et al., Highly sensitive gamma-spectrometers of gerda for material screening: Part I (2008). arXiv:0812.0723

25. D. Budjas et al., Highly sensitive gamma-spectrometers of gerda for material screening: Part II (2008). arXiv:0812.0768

26. ILIAS. Ilias database on radiopurity of materials. http://radiopurity. in $2 \mathrm{p} 3 . \mathrm{fr} /$

27. K. Abe et al., Nucl. Instrum. Methods Phys. Res. Sect. A Accel. Spectrom. Detect. Assoc. Equip. 884, 157 (2018). https://doi.org/ 10.1016/j.nima.2017.12.015

28. J. Kaizer, S. Nisi, P. Povinec, J. Radioanal. Nucl. Chem. 322(3), 1447 (2019). https://doi.org/10.1007/s10967-019-06857-3

29. D. Unger et al., J. Instrum. 16(06), P06006 (2021). https://doi.org/ 10.1088/1748-0221/16/06/p06006

30. Naturally Occurring Radioactive Materials (NORM IV) Proceedings of an International Conference held in Szczyrk, Poland, 17-21 May 2004. No. 1472 in TECDOC Series (INTERNATIONAL ATOMIC ENERGY AGENCY, Vienna, 2005). https://www.iaea.org/publications/7384/naturally-occurri ng-radioactive-materials-norm-iv-proceedings-of-an-international -conference-held-in-szczyrk-poland-17-21-may-2004
31. J.P. McBride et al., Science 202(4372), 1045 (1978). https://doi. org/10.1126/science.202.4372.1045. https://science.sciencemag. org/content $/ 202 / 4372 / 1045$

32. T.R. Ajayi et al., Environ. Geochem. Health 31(1), 61 (2009). https://doi.org/10.1007/s10653-008-9155-z

33. S. Agostinelli et al., Nucl. Instrum. Methods Phys. Res. Sect. A Accel. Spectrom. Detect. Assoc. Equip. 506(3), 250 (2003). https:// doi.org/10.1016/S0168-9002(03)01368-8

34. O. Palais, E. Yakimov, S. Martinuzzi, Mater. Sci. Eng. B 91-92, 216 (2002). https://doi.org/10.1016/S0921-5107(01)00998-9

35. S. Dubois et al., J. Appl. Phys. 102(8), 083525 (2007). https://doi. org/10.1063/1.2799057

36. L. Landau, J. Phys. (USSR) 8, 201 (1944)

37. D. Wilkinson, Nucl. Instrum. Methods Phys. Res. Sect. A Accel. Spectrom. Detect. Assoc. Equip. 383(2), 513 (1996). https://doi. org/10.1016/S0168-9002(96)00774-7

38. A. Göggelmann, Ph.D. thesis, University Tuebingen (in preparation) 